Supporting Information for

\title{
Efficient Palladium-Catalyzed Cross-Coupling of $\beta$-chloro- alkylidene/arylidene Malonates using Microwave Chemistry
}

\section{Rajamohan R. Poondra, Peter M. Fischer and Nicholas J. Turner *}

School of Chemistry, University of Edinburgh, Kings Buildings, West Mains Road, Edinburgh, EH9 3JJ, UK and Cyclacel Ltd, James Lindsay Place, Dundee DD1 5JJ, $U K$

Contents

General experimental information $\quad$ S2

General procedure for cross-coupling reaction $\quad$ S3

$\begin{array}{ll}\text { Characterization data of compound (entry 1) S3 } & \text { S3 }\end{array}$

Characterization data of compounds (entry 2\&3) S4

Characterization data of compounds (entry 4\&5) S5

Characterization data of compounds (entry 6\&7) S6

$\begin{array}{ll}\text { Characterization data of compounds (entry 8\&9) } & \text { S7 }\end{array}$

Characterization data of compounds (entry 10\&11) S8

Characterization data of compounds (entry 12\&13) S9

$\begin{array}{ll}\text { Characterization data of compounds (entry 14\&15) } & \text { S10 }\end{array}$

${ }^{1} \mathrm{H}$ NMR \& ${ }^{13} \mathrm{C}$ NMR spectra of compound (entry1) S11

$\begin{array}{ll}{ }^{1} \mathrm{H} \text { NMR } \&{ }^{13} \mathrm{C} \text { NMR spectra of compound (entry3) } & \mathrm{S} 12\end{array}$

$\begin{array}{ll}{ }^{1} \mathrm{H} \text { NMR spectra of compound (entry2) } & \mathrm{S} 13\end{array}$

$\begin{array}{ll}{ }^{1} \mathrm{H} \text { NMR \& }{ }^{13} \mathrm{C} \text { NMR spectra of compound (entry 4) } & \text { S14 }\end{array}$

$\begin{array}{ll}{ }^{1} \mathrm{H} \text { NMR spectra of compounds (entry 5\&6) } & \text { S15 }\end{array}$

$\begin{array}{ll}{ }^{1} \mathrm{H} \text { NMR spectra of compounds (entry 7\&8) } & \text { S16 }\end{array}$

${ }^{1} \mathrm{H}$ NMR \& ${ }^{13} \mathrm{C}$ NMR spectra of compound (entry 9) $\quad$ S17

$\begin{array}{ll}{ }^{1} \mathrm{H} \text { NMR spectra of compounds (entry 10\&11) } & \text { S18 }\end{array}$

$\begin{array}{ll}{ }^{1} \mathrm{H} \text { NMR \& }{ }^{13} \mathrm{C} \text { NMR spectra of compound (entry 12) } & \text { S19 }\end{array}$

${ }^{1} \mathrm{H}$ NMR \& ${ }^{13} \mathrm{C}$ NMR spectra of compound (entry 13) $\quad$ S20

${ }^{1} \mathrm{H}$ NMR \& ${ }^{13} \mathrm{C}$ NMR spectra of compound (entry 14) $\quad$ S21

${ }^{1} \mathrm{H}$ NMR \& ${ }^{13} \mathrm{C}$ NMR spectra of compound (entry 15) S22 


\section{General experimental information:}

Unless otherwise noted, all materials were obtained from commercial suppliers and used without further purification. THF was dried and distilled from sodium/benzophenone. $\mathrm{K}_{2} \mathrm{CO}_{3}$ was purchased dry from commercial suppliers and dried under vacuum at $120{ }^{\circ} \mathrm{C}$ for $24 \mathrm{~h}$. Thin-Layer Chromatography was performed on precoated plates, silica gel $60 \mathrm{~F}_{254}$ and visualizing with ultraviolet light and iodine spray. Flash chromatography on silica gel. All melting points are uncorrected. Infrared spectra were observed as $\mathrm{KBr}$ pellets or neat. All ${ }^{1} \mathrm{H}$ NMR and ${ }^{13} \mathrm{C}$ NMR spectra were recorded in $\mathrm{CDCl}_{3}$ and $\mathrm{DMSO}-\mathrm{d}_{6}$ solutions using the residual solvent peak as internal reference. Spin multiplicities are given as s (singlet), $d$ (doublet), $t$ (triplet), q (quartet), and m (multiplet) as well as b (broad). Coupling constants (J) are given in hertz. Mass spectra Electro spray and HRMS were obtained.

All microwave irradiation experiments were carried out using the Explorer PLS. ${ }^{\mathrm{TM}}$ The reactions were performed in heavy-walled Pyrex tubes (10 ml, $1=150 \mathrm{~mm})$ sealed with a septum utilizing the standard absorbance level (300 W maximum power). The reaction volume filled not more than $14^{\text {th }}$ of the total volume of the tube. All couplings and other reactions were conducted in the presence of stirring and external cooling. 


\section{General Procedure for the Reaction of $\beta$-chloro-arylidene/alkylidenemalonates with arylboronic acids:}

POPd (1mol \%), $\beta$-Chloro-alkylidene/arylidenemalonate (1.00 mmol), arylboronic acid (1.50 to $2.00 \mathrm{mmol})$, and $\mathrm{K}_{2} \mathrm{CO}_{3}(3.00 \mathrm{mmol})$ were weighed in a microwave tube, equipped with a magnetic stirrer bar, and sealed with a silicon septum. THF (2 to 3 $\mathrm{mL}$ ) was injected into the tube via a syringe and the reaction mixture was subjected to microwave irradiation for $30 \mathrm{~min}$ at $100{ }^{\circ} \mathrm{C}$.The reaction vessel was allowed to cool to room temperature and the crude reaction mixture transferred to a separating funnel and diluted with hexane $(50 \mathrm{~mL})$ and $\mathrm{H}_{2} \mathrm{O}(15 \mathrm{~mL})$. The layers were separated, the organic layer was washed with $\mathrm{H}_{2} \mathrm{O}(20 \mathrm{~mL})$ and brine $(20 \mathrm{~mL})$, dried over $\mathrm{MgSO}_{4}$, and filtered, and solvents were removed from the filtrate by rotary evaporation. The resulting residue was chromatographed on silica gel using ethyl acetate/hexane as eluant.

\section{2-[1-(3-Chlorophenyl) ethylidene] malonic acid diethyl ester (Entry 1):}

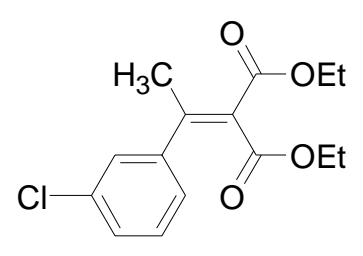

2-[1-(3-Chlorophenyl) ethylidene] malonic acid diethyl ester was prepared using the general procedure. POPd (12 mg, $0.023 \mathrm{mmol}, 1 \% \mathrm{~mol}), 2$-(1-Chloro-ethylidene)malonic acid diethyl ester ( $0.5 \mathrm{~g}, 2.26 \mathrm{mmol})$, 3-chlorophenyl boronic acid (0.532 g, $3.40 \mathrm{mmol})$, and $\mathrm{K}_{2} \mathrm{CO}_{3}(0.940 \mathrm{~g}, 6.80 \mathrm{mmol})$ in THF $(3 \mathrm{~mL})$ yielded $0.470 \mathrm{~g}$ (70\% yield) of the title product as a colorless liquid. $R_{f}=0.5$ (hexane/ethyl acetate $=9: 1$ ); IR (Neat): 2982, 1725, 1628, 1563, 1473, 1445, $1228 \mathrm{~cm}^{-1} .{ }^{1} \mathrm{H}$ NMR (250 MHz, $\mathrm{CDCl}_{3}$ ): $\delta 7.45$ (m, 3H), 7.31 (s, 1H), 4.49 (q, $\left.J=7.14 \mathrm{~Hz}, 2 \mathrm{H}\right), 4.20$ (q, $J=7.15 \mathrm{~Hz}$, 2H), 2.59 (s, 3H), 1.51 (t, $J=7.13 \mathrm{~Hz}, 3 \mathrm{H}), 1.21$ (t, $J=7.13 \mathrm{~Hz}, 3 \mathrm{H}) .{ }^{13} \mathrm{C}$ NMR (63 $\mathrm{MHz}_{\mathrm{CDCl}}$ ): $\delta 165.5,164.4,153.5,143.1,134.1,129.5,128.3,126.7,124.7,61.8$, 22.5, 13.9. HRMS (EI $\left.{ }^{+}\right): \mathrm{m} / \mathrm{z}$ calculated for $\mathrm{C}_{15} \mathrm{H}_{17} \mathrm{ClO}_{4}$, 296.0815; found, 296.0813. 
2-[1-(3, 5-Difluoro phenyl) ethylidene] malonic acid diethyl ester (Entry 2):

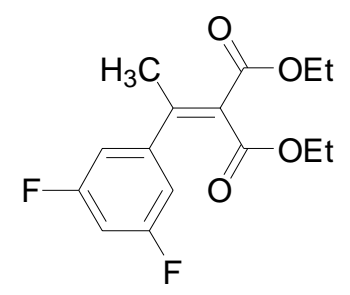

2-[1-(3,5-Difluoro phenyl) ethylidene] malonic acid diethyl ester was prepared using the general procedure. POPd, (11.3 mg, $0.02 \mathrm{mmol}, 1 \% \mathrm{~mol})$, 2-(1-Chloroethylidene)-malonic acid diethyl ester (0.5 g, $2.26 \mathrm{mmol})$, 3,5-fluorophenyl boronic acid (0.716 g, $4.53 \mathrm{mmol})$, and $\mathrm{K}_{2} \mathrm{CO}_{3}(0.940 \mathrm{~g}, 6.80 \mathrm{mmol})$ in THF (3mL) yielded 0.4 g (59 \% yield) of the title product as a colorless liquid. $R_{f}=0.4$ (hexane/ethyl acetate = 8:2); IR (Neat): 2984, 1726, 1641, 1620, 1590, 1492, 1446, $1236 \mathrm{~cm}^{-1} .{ }^{1} \mathrm{H}$ NMR (250 MHz, CDCl ${ }_{3}$ ): $\delta 7.25$ (m, 2H), $7.11(\mathrm{~m}, 1 \mathrm{H}), 4.55$ (q, $\left.J=7.12 \mathrm{~Hz}, 2 \mathrm{H}\right), 4.25$ (q, $J$ $=7.12 \mathrm{~Hz}, 2 \mathrm{H}), 2.57(\mathrm{~s}, 3 \mathrm{H}), 1.58(\mathrm{t}, J=7.11 \mathrm{~Hz}, 3 \mathrm{H}), 1.28(\mathrm{t}, J=7.12 \mathrm{~Hz}, 3 \mathrm{H})$. HRMS (EI ${ }^{+}$): m/z calculated for $\mathrm{C}_{15} \mathrm{H}_{16} \mathrm{~F}_{2} \mathrm{O}_{4}$, 298.1017; found, 298.1020.

\section{2-[1-(3, 5-Dimethyl phenyl) ethylidene] malonic acid diethyl ester (Entry 3):}

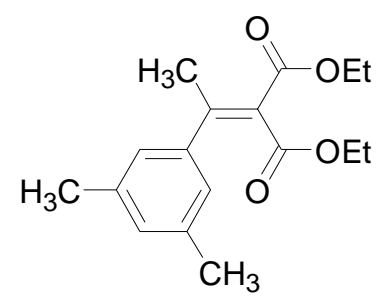

2-[1-(3,5-Dimethyl phenyl) ethylidene] malonic acid diethyl ester was prepared using the general procedure. POPd, (6 mg, $0.011 \mathrm{mmol}, 1$ \% mol), 2-(1-Chloro-ethylidene)malonic acid diethyl ester (0.250 g, $1.13 \mathrm{mmol})$, 3,5-methylphenyl boronic acid (0.255 g, $1.70 \mathrm{mmol})$, and $\mathrm{K}_{2} \mathrm{CO}_{3}(0.470 \mathrm{~g}, 3.40 \mathrm{mmol})$ in THF (2mL) yielded $0.2 \mathrm{~g}$ (60\% yield) of the title product as a colorless liquid. $R_{f}=0.5$ (hexane/ethyl acetate $=$ 9:1); IR (Neat): 2981, 1724, 1623, 1445, 1266, $1223 \mathrm{~cm}^{-1} .{ }^{1} \mathrm{H}$ NMR (250 MHz, $\mathrm{CDCl}_{3}$ ): $\delta 7.11$ (s, 1H), 7.01 (s, 2H), 4.43 (q, $\left.J=7.12 \mathrm{~Hz}, 2 \mathrm{H}\right), 4.15$ (q, $J=7.13 \mathrm{~Hz}$, 2H), 2.56 (s, 3H), 2.45 (s, 6H) 1.47 (t, $J=7.11 \mathrm{~Hz}, 3 \mathrm{H}), 1.15$ (t, $J=7.12 \mathrm{~Hz}, 3 \mathrm{H}) .{ }^{13} \mathrm{C}$ NMR (63 MHz, $\left.\mathrm{CDCl}_{3}\right): \delta 166.2,164.6,156.0,141.4,137.6,129.9,125.7,124.2$, 60.8, 22.6, 13.9. HRMS $\left(\mathrm{EI}^{+}\right): \mathrm{m} / \mathrm{z}$ calculated for $\mathrm{C}_{17} \mathrm{H}_{12} \mathrm{O}_{4}, 290.1518$; found, 290.1520 . 
2-[1-(4-Methoxyphenyl)-ethylidene]-malonic acid diethyl ester (Entry 4):

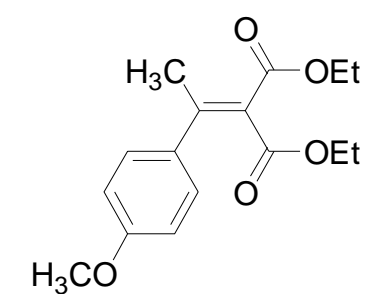

2-[1-(4-Methoxyphenyl)-ethylidene]-malonic acid diethyl ester was prepared using the general procedure. POPd, (6 mg, $0.011 \mathrm{mmol}$, $0.1 \% \mathrm{~mol})$, 2-(1-Chloroethylidene)-malonic acid diethyl ester (0.250 g, $1.13 \mathrm{mmol})$, 4-methoxyphenyl boronic acid (0.258 g, 1.70mmol), and $\mathrm{K}_{2} \mathrm{CO}_{3}(0.470 \mathrm{~g}, 3.40 \mathrm{mmol})$ in THF (3mL) yielded $0.210 \mathrm{~g}$ (65\% yield) of the title product as a colorless liquid. $R_{f}=0.5$ (hexane/ethyl acetate $=8: 2) .{ }^{1} \mathrm{H}$ NMR $\left(250 \mathrm{MHz}, \mathrm{CDCl}_{3}\right): \delta 7.13(\mathrm{~d}, \mathrm{~J}=8.48 \mathrm{~Hz}$, 2H), 6.78 (d, $J=8.57 \mathrm{~Hz}, 2 \mathrm{H}), 4.50$ (q, $J=7.10 \mathrm{~Hz}, 2 \mathrm{H}), 3.94$ (q, J = 7.10 Hz, 2H), 3.73 (s, 3H), 2.35 (s, 3H), 1.25 (t, $J=7.11 \mathrm{~Hz}, 3 \mathrm{H}), 0.97$ (t, J = $7.12 \mathrm{~Hz}, 3 \mathrm{H}) .{ }^{13} \mathrm{C}$ NMR (63 MHz, $\left.\mathrm{CDCl}_{3}\right): \delta 166.5,164.7,159.7,155.4,133.6,128.1,125.4,113.5$, 60.8, 55.1, 22.6, 13.9. MS (ES $\left.{ }^{+}\right):$m/z (relative intensity) 292(M+, 15), 260 (15), 172 (20), 110 (55), 97 (100), 80 (15).

\section{2-[1-(5-Fluoro-2-methoxyphenyl) ethylidene]malonic acid diethyl ester (Entry 5):}

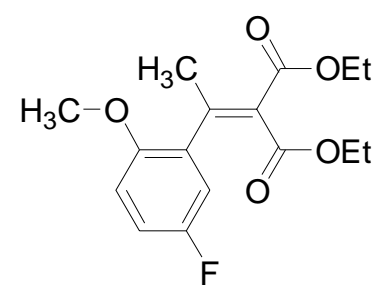

2-[1-(5-Fluoro-2-methoxyphenyl) ethylidene] malonic acid diethyl ester was prepared using the general procedure. POPd, (11.5 mg, $0.02 \mathrm{mmol}, 1 \% \mathrm{~mol})$, 2-(1-Chloroethylidene)-malonic acid diethyl ester (0.5 g, $2.26 \mathrm{mmol})$, 2-methoxy-5-fluorophenyl boronic acid $(0.770 \mathrm{~g}, 4.53 \mathrm{mmol})$, and $\mathrm{K}_{2} \mathrm{CO}_{3}(0.940 \mathrm{~g}, 6.80 \mathrm{mmol})$ in THF (3mL) yielded $0.280 \mathrm{~g}$ (66 \% yield) of the title product as a colorless liquid. $R_{f}=0.5$ (hexane/ethyl acetate =9:1); IR (Neat): 2982, 1725, 1637, 1610, 1594, $1465 \mathrm{~cm}^{-1}$. ${ }^{1} \mathrm{H}$ NMR (250 MHz, $\left.\mathrm{CDCl}_{3}\right): \delta 7.27(\mathrm{~m}, 3 \mathrm{H}), 4.55$ (q, $\left.J=7.11 \mathrm{~Hz}, 2 \mathrm{H}\right), 4.27$ (q, $J=$ $7.15 \mathrm{~Hz}, 2 \mathrm{H}), 4.06$ (s, 3H), 2.60 (s, 3H), 1.57 (t, J= $7.15 \mathrm{~Hz}, 3 \mathrm{H}), 1.23$ (t, J = 7.14 Hz, 3H). HRMS (EI $\left.{ }^{+}\right): \mathrm{m} / \mathrm{z}$ calculated for $\mathrm{C}_{16} \mathrm{H}_{19} \mathrm{FO}_{5}, 310.1217$; found 310.1216. 


\section{2-[-(3-Nitrophenyl) ethylidene] malonic acid diethyl ester (Entry 6):}

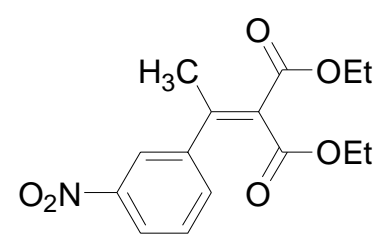

2-[-(3-Nitrophenyl) ethylidene] malonic acid diethyl ester was prepared using the general procedure. POPd, (11.3 mg, $0.02 \mathrm{mmol}, 1 \% \mathrm{~mol})$, 2-(1-Chloro-ethylidene)malonic acid diethyl ester (0.5 g, $2.26 \mathrm{mmol})$, 3-nitrophenyl boronic acid (0.757g, $4.53 \mathrm{mmol})$, and $\mathrm{K}_{2} \mathrm{CO}_{3}(0.940 \mathrm{~g}, 6.80 \mathrm{mmol})$ in THF $(3 \mathrm{~mL})$ yielded $0.3 \mathrm{~g}(72 \%$ yield) of the title product as a colorless liquid. $R_{f}=0.3$ (hexane/ethyl acetate $=9: 1$ ); IR (Neat): 2984, 1724, 1631, 1574, 1474, $1446 \mathrm{~cm}^{-1} .{ }^{1} \mathrm{H}$ NMR (250 MHz, $\left.\mathrm{CDCl}_{3}\right): \delta$ 8.12 (m, 1H), 8.05 (s, 1H), 7.48 (m, 2H), 4.25 (q, $J=7.17 \mathrm{~Hz}, 2 \mathrm{H}), 3.92$ (q, $J=7.13$ Hz, 2H), 2.33 (s, 3H), 1.29 (t, $J=7.09 \mathrm{~Hz}, 3 \mathrm{H}$ ), 0.97 (t, $J=7.11 \mathrm{~Hz}, 3 \mathrm{H}$ ). HRMS $\left(\mathrm{EI}^{+}\right)$: m/z calculated for $\mathrm{C}_{15} \mathrm{H}_{17} \mathrm{NO}_{6}, 307.1056$; found, 307.1049.

\section{2-(2-Methyl-1-phenylpropylidene) malonic acid diethyl ester (Entry 7):}

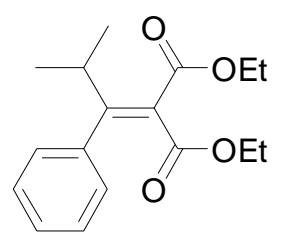

2-(2-Methyl-1-phenylpropylidene) malonic acid diethyl ester was prepared using the general procedure. POPd, (10 mg, 0.012 mmol, 1 \% mol), 2-(1-Chloro-2-methyl propylidene) malonic acid diethyl ester (0.5g, $2.0 \mathrm{mmol})$, 3-phenyl boronic acid (0.294 g, $2.40 \mathrm{mmol})$, and $\mathrm{K}_{2} \mathrm{CO}_{3}(0.834 \mathrm{~g}, 6.03 \mathrm{mmol})$ in THF (3mL) yielded $0.250 \mathrm{~g}$ (71 \% yield) of the title product as a colorless liquid. $R_{f}=0.4$ (hexane/ethyl acetate = 9:1). ${ }^{1} \mathrm{H}$ NMR (250 MHz, $\mathrm{CDCl}_{3}$ ): $\delta 7.37$ (m, 3H), 7.12 (m, 2H), 4.40 (q, $J=7.14 \mathrm{~Hz}$, 2H), 3.94 (q, $J=7.17 \mathrm{~Hz}, 2 \mathrm{H}), 3.57$ (m, 1H), 1.40 (t, $J=7.14 \mathrm{~Hz}, 3 \mathrm{H}), 1.07$ (d, $J=$ $6.84 \mathrm{~Hz}, 6 \mathrm{H}), 0.99$ (t, $J=7.14 \mathrm{~Hz}, 3 \mathrm{H}) . \mathrm{MS}\left(\mathrm{ES}^{+}\right): \mathrm{m} / \mathrm{z}$ (relative intensity) $313(\mathrm{M}+$ Na, 100), 240 (5), 163 (25). 
2-[1-(3-Chlorophenyl)-2-methylpropylidene] malonic acid diethyl ester (Entry 8):

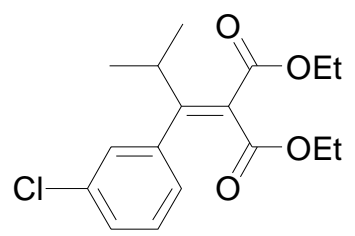

2-[1-(3-Chlorophenyl)-2-methylpropylidene] malonic acid diethyl ester was prepared using the general procedure. POPd, (6 mg, $0.012 \mathrm{mmol}$, 1\% mol), 2-(1-Chloro-2methyl propylidene) malonic acid diethyl ester (0.3g, $1.20 \mathrm{mmol})$, 3-chlorophenyl boronic acid (0.283 g, $0.181 \mathrm{mmol}) \mathrm{K}_{2} \mathrm{CO}_{3}(0.5 \mathrm{~g}, 3.62 \mathrm{mmol})$ in THF (2mL) yielded ( $0.15 \mathrm{~g}, 57 \%$ yield) of the title product as a colorless liquid. IR (Neat): 2978, 1726, 1624, 1563, 1466, $1446 \mathrm{~cm}^{-1} .{ }^{1} \mathrm{H}$ NMR (250 MHz, $\left.\mathrm{CDCl}_{3}\right): \delta 7.45$ (m, 2H), $7.26(\mathrm{~m}$, 1H), 7.14 (m, 1H), 4.49 (q, $J=3.45 \mathrm{~Hz}, 2 \mathrm{H}), 4.07$ (q, $J=7.14 \mathrm{~Hz}, 2 \mathrm{H}), 3.60(\mathrm{~m}, 1 \mathrm{H})$, 1.52 (t, $J=3.74 \mathrm{~Hz}, 3 \mathrm{H}), 1.20$ (s, 3H), 1.87 (s, 3H), 1.13 (t, $J=7.14 \mathrm{~Hz}, 3 \mathrm{H}$ ); HRMS $\left(\mathrm{EI}^{+}\right): \mathrm{m} / \mathrm{z}$ calculated for $\mathrm{C}_{17} \mathrm{H}_{21} \mathrm{ClO}_{4}$, 324.1128; found, 324.1297.

\section{2-[1-(3-Fluoro phenyl)-2-methylpropylidene] malonic acid diethyl ester(Entry 9):}

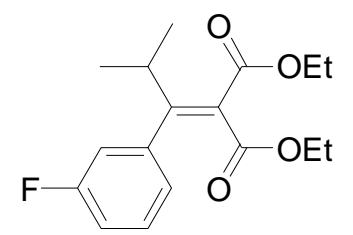

2-[1-(3-Fluoro phenyl)-2-methyl propylidene] malonic acid diethyl ester was prepared using the general procedure. POPd, (10 mg, $0.02 \mathrm{mmol}, 1 \% \mathrm{~mol})$, 2-(1Chloro-2-methyl propylidene) malonic acid diethyl ester (0.5 g, $2.0 \mathrm{mmol}$ ), 3fluorophenyl boronic acid (0.337 g, $2.40 \mathrm{mmol})$, and $\mathrm{K}_{2} \mathrm{CO}_{3}(0.834 \mathrm{~g}, 6.0 \mathrm{mmol})$ in THF (3mL) yielded $0.340 \mathrm{~g}$ (55\% yield) of the title product as a colorless liquid. $R_{f}=$ 0.6 (hexane/ethyl acetate $=9: 1) .{ }^{1} \mathrm{H}$ NMR $\left(250 \mathrm{MHz}, \mathrm{CDCl}_{3}\right): \delta 7.28(\mathrm{~m}, 1 \mathrm{H}), 7.02$ (m, 1H), 6.84 (m, 2H), 4.30 (q, $J=7.13 \mathrm{~Hz}, 2 \mathrm{H}), 3.92$ (q, $J=7.13 \mathrm{~Hz}, 2 \mathrm{H}), 3.46$ (m, 1H), 1.04 (d, $J=7.13 \mathrm{~Hz}, 6 \mathrm{H}), 0.96$ (t, $J=7.14 \mathrm{~Hz}, 3 \mathrm{H}) .{ }^{13} \mathrm{C}$ NMR $\left(63 \mathrm{MHz}, \mathrm{CDCl}_{3}\right)$ : $\delta$ 164.8, 163.8, 161.4, 159.9, 138.3, 129.0, 126.1, 123.6, 115.3, 61.2, 31.9, 20.5, 13.9 . MS (ES $\left.{ }^{+}\right):$m/z (relative intensity) 331(M+, +Na, 5), 300 (40), 198 (18), 130 (100), 73 (10), 61(50). 


\section{2-[1-(5-Fluoro-2-methoxyphenyl)-2-methylpropylidene] malonic acid diethyl ester (Entry 10):}

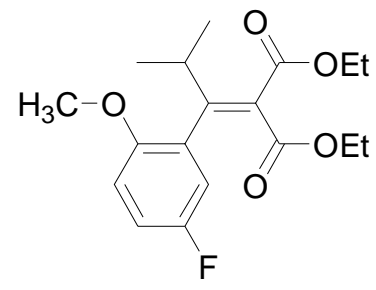

2-[1-(5-Fluoro-2-methoxyphenyl)-2-methylpropylidene] malonic acid diethyl ester was prepared using the general procedure. POPd, (10 mg, $0.019 \mathrm{mmol}, 1 \% \mathrm{~mol}$ ), 2(1-Chloro-2-methyl propylidene) malonic acid diethyl ester (0.5 g, $2.0 \mathrm{mmol}), 2-$ methoxy-5-fluorophenyl boronic acid (0.684 g, $4.0 \mathrm{mmol})$, and $\mathrm{K}_{2} \mathrm{CO}_{3}(0.830 \mathrm{~g}, 6.0$ $\mathrm{mmol}$ ) in THF (3mL) yielded $0.250 \mathrm{~g}$ (52 \% yield) of the title product as a colorless liquid. $R_{f}=0.5$ (hexane/ethyl acetate = 9:1); IR (Neat): 2979, 1726, 1628, 1607, 1495, $1465 \mathrm{~cm}^{-1} .{ }^{1} \mathrm{H}$ NMR (250 MHz, $\left.\mathrm{CDCl}_{3}\right): \delta 7.18(\mathrm{~m}, 1 \mathrm{H}), 6.98(\mathrm{~m}, 1 \mathrm{H}), 6.88(\mathrm{~m}, 1 \mathrm{H})$, 4.51 (m, 2H), 4.11 (q, $J=7.16 \mathrm{~Hz}, 2 \mathrm{H}), 3.96$ (s, 3H), 3.57 (m, 1H), 1.35 (t, $J=7.14$ $\mathrm{Hz}, 3 \mathrm{H}), 1.21(\mathrm{~m}, 9 \mathrm{H})$. HRMS $\left(\mathrm{EI}^{+}\right): \mathrm{m} / \mathrm{z}$ calculated for $\mathrm{C}_{18} \mathrm{H}_{23} \mathrm{FO}_{5}, 338.1530$; found, 338.1528 .

\section{2-[(3-Chloro-phenyl)-phenylmethylene] malonic acid diethyl ester (Entry 11):}

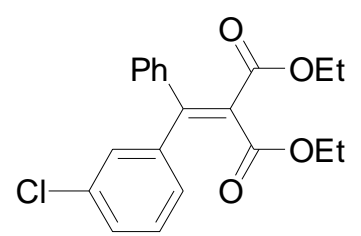

2-[(3-Chloro-phenyl)-phenylmethylene] malonic acid diethyl ester was prepared using the general procedure. POPd, (3.5 mg, $0.0069 \mathrm{mmol}, 1 \% \mathrm{~mol}$ ), 2-(Chlorophenyl methylene) malonic acid diethyl ester (0.2 g, 0.707mmol), 3-chlorophenyl boronic acid (0.166 g, $1.06 \mathrm{mmol}$ ), and $\mathrm{K}_{2} \mathrm{CO}_{3}(0.293 \mathrm{~g}, 2.12 \mathrm{mmol})$ in THF (2mL) yielded $0.14 \mathrm{~g}$ (55\% yield) of the title product as a colorless liquid. $R_{f}=0.5$ (hexane/ethyl acetate = 9:1); IR (Neat): 2982, 1728, 1591, 1472, $1444 \mathrm{~cm}^{-1} .{ }^{1} \mathrm{H}$ NMR (250 MHz, $\left.\mathrm{CDCl}_{3}\right): \delta 7.18(\mathrm{~m}, 9 \mathrm{H}), 4.04(\mathrm{~m}, 4 \mathrm{H}), 0.97(\mathrm{~m}, 6 \mathrm{H}) . \mathrm{HRMS}\left(\mathrm{EI}^{+}\right): \mathrm{m} / \mathrm{z}$ calculated for $\mathrm{C}_{20} \mathrm{H}_{19} \mathrm{ClO}_{4}$, 358.0972; found, 358.0975. 


\section{2-[(5-Fluoro-2-methoxyphenyl) phenylmethylene] malonic acid diethyl ester} (Entry 12):

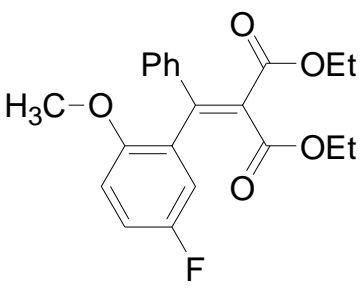

2-[(5-Fluoro-2-methoxyphenyl) phenylmethylene] malonic acid diethyl ester was prepared using the general procedure. POPd, (9 mg, $0.017 \mathrm{mmol}$ 1\%mol), 2-(Chlorophenyl methylene) malonic acid diethyl ester (0.5 g, 1.76), 2-methoxy-5-fluorophenyl boronic acid (0.601 g, $3.53 \mathrm{mmol})$, and $\mathrm{K}_{2} \mathrm{CO}_{3}(0.735 \mathrm{~g}, 5.31 \mathrm{mmol})$ in THF (3mL) yielded $0.2 \mathrm{~g}$ (50\% yield) of the title product as a colorless liquid. $R_{f}=0.5$ (hexane/ethyl acetate $=8.5: 1.5$ ); IR (Neat): 2982, 1728, 1619, 1591, $1494 \mathrm{~cm}^{-1} .{ }^{1} \mathrm{H}$ NMR (250 MHz, $\mathrm{CDCl}_{3}$ ): $\delta 7.17$ (m, 6H), 6.72 (m, 2H), 3.97 (m, 4H), 3.59 (s, 3H), 1.01 (m, 6H). ${ }^{13} \mathrm{C}$ NMR (63 MHz, $\left.\mathrm{CDCl}_{3}\right): \delta 166.0,164.6,158.4,154.6,152.5,150.5$, 139.5, 130.9, 128.8, 128.1, 126.7, 116.6, 112.1, 60.2, 56.1, 13.6. HRMS (EI $\left.{ }^{+}\right): \mathrm{m} / \mathrm{z}$ calculated for $\mathrm{C}_{21} \mathrm{H}_{21} \mathrm{FO}_{5}$, 372.1373; found 372.1368.

\section{2-[phenyl- (3-trifluoromethyl-phenyl)-methylene] malonic acid diethyl ester} (Entry 13):

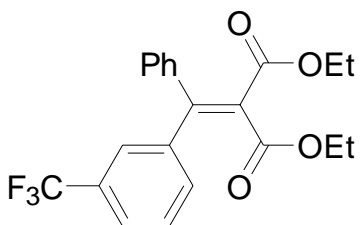

2-[phenyl- (3-trifluoromethyl-phenyl)-methylene] malonic acid diethyl ester was prepared using the general procedure. POPd, (3.5 mg, $0.0070 \mathrm{mmol}, 1 \% \mathrm{~mol}$ ), 2(Chloro-phenyl methylene) malonic acid diethyl ester (0.2 g, $0.707 \mathrm{mmol}), 3-$ trifluorophenyl boronic acid (0.161 g, $0.84 \mathrm{mmol})$, and $\mathrm{K}_{2} \mathrm{CO}_{3}(0.29 \mathrm{~g}, 2.12 \mathrm{mmol})$ in THF ( $2 \mathrm{~mL}$ ) yielded $0.19 \mathrm{~g}$ (68\% yield) of the title product as a pale brown solid. $R_{f}=$ 0.3 (hexane/ethyl acetate $=9.5: 0.5), \mathrm{mp}=58-59.5{ }^{0} \mathrm{C} . \mathrm{IR}(\mathrm{KBr}): 2980,1721,1698$, 1604, $1444 \mathrm{~cm}^{-1} .{ }^{1} \mathrm{H}$ NMR (250 MHz, $\left.\mathrm{CDCl}_{3}\right): \delta 7.64(\mathrm{~m}, 9 \mathrm{H}), 4.27(\mathrm{~m}, 4 \mathrm{H}), 1.21(\mathrm{~m}$, $6 \mathrm{H}) .{ }^{13} \mathrm{C}$ NMR $\left(63 \mathrm{MHz}, \mathrm{CDCl}_{3}\right): \delta$ 153.2, 140.1, 138.5, 131.8, 128.9, 128.4, 128.1, 127.7, 125.1, 76.9, 76.4, 75.9, 60.9, 12.9. HRMS (FAB): $\mathrm{m} / \mathrm{z}$ calculated for $\mathrm{C}_{21} \mathrm{H}_{19} \mathrm{~F}_{3} \mathrm{O}_{4}$, 392.1235; found, 392.1346. 


\section{2-[(3-Amino-phenyl)-phenylmethylene] malonic acid diethyl ester (Entry 14):}

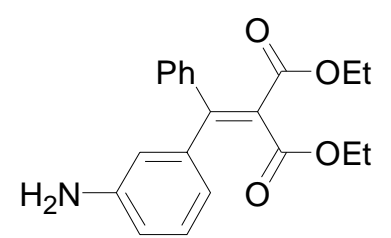

2-[(3-Amino-phenyl)-phenylmethylene] malonic acid diethyl ester was prepared using the general procedure. POPd, (9 mg, $0.017 \mathrm{mmol}$, 1\% mol), 2-(Chloro-phenyl methylene) malonic acid diethyl ester ( $0.5 \mathrm{~g}, 1.76 \mathrm{mmol}), 3$-aminophenyl boronic acid (0.493 g, $2.65 \mathrm{mmol}$ ), and $\mathrm{K}_{2} \mathrm{CO}_{3}(0.733 \mathrm{~g}, 5.30 \mathrm{mmol})$ in THF (3mL) yielded $0.250 \mathrm{~g}$ (60\% isolated yield) of the title product as a light brown solid. $R_{f}=0.5$ (hexane/ethyl acetate $=7: 3), \mathrm{mp}=73-74{ }^{0} \mathrm{C} . \mathrm{IR}(\mathrm{KBr}):$ 3462, 3372, 2988, 1731, 1597, 1488, 1445 $\mathrm{cm}^{-1} .{ }^{1} \mathrm{H}$ NMR (250 MHz, $\left.\mathrm{CDCl}_{3}\right): \delta 7.14(\mathrm{~m}, 6 \mathrm{H}), 6.46(\mathrm{~m}, 2 \mathrm{H}), 6.37(\mathrm{~s}, 1 \mathrm{H}), 4.00(\mathrm{~m}$, 4H), 3.56(bs, 2H), 0.95 (m, 6H). ${ }^{13} \mathrm{C}$ NMR (63 MHz, $\left.\mathrm{CDCl}_{3}\right): \delta 166.0,165.8,155.7$, 146.0, 141.0, 139.8, 128.9, 127.9, 125.9, 119.3, 115.7, 115.4, 61.0, 13.5. HRMS (FAB): $\mathrm{m} / \mathrm{z}$ calculated for $\mathrm{C}_{20} \mathrm{H}_{21} \mathrm{NO}_{4}, 339.1471$; found, 339.1468 .

\section{2-[(3-Hydroxy-phenyl)-phenyl methylene] malonic acid diethyl ester (Entry 15):}

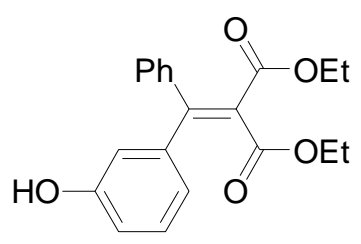

2-[(3-Hydroxy-phenyl)-phenyl methylene] malonic acid diethyl ester was prepared using the general procedure. POPd, (9 mg, $0.017 \mathrm{mmol}, 1 \% \mathrm{~mol})$, 2-(Chloro-phenyl methylene) malonic acid diethyl ester (0.5 g, $1.76 \mathrm{mmol})$, 3-hydroxyphenyl boronic acid (0.366 g, $2.65 \mathrm{mmol}$ ), and $\mathrm{K}_{2} \mathrm{CO}_{3}(0.733 \mathrm{~g}, 5.30 \mathrm{mmol}$ ) in THF (3mL) yielded $0.380 \mathrm{~g}$ (63\% yield) of the title product as a white colour solid. $R_{f}=0.5$ (hexane/ethyl acetate $=7: 3), \mathrm{mp}=94-95.5{ }^{0} \mathrm{C} . \mathrm{IR}(\mathrm{KBr}): 3389,2989,1707,1673,1578,1444 \mathrm{~cm}^{-1}$. ${ }^{1} \mathrm{H}$ NMR (250 MHz, CDCl $)$ ): $\delta 7.32$ (m, 6H), 6.66 (m, 2H), 6.43(s, 1H), 5.70(bs, 1H), 4.14(m, 2H), 1.10(m, 6H). ${ }^{13} \mathrm{C}$ NMR (63 $\left.\mathrm{MHz} \mathrm{CDCl}_{3}\right): \delta 166.3,155.6,141.2,139.6$, 129.2, 129.1, 129.0, 128.0, 121.2, 116.3, 61.2, 13.5. HRMS (FAB): m/z calculated for $\mathrm{C}_{20} \mathrm{H}_{20} \mathrm{O}_{5}, 340.1311$; found, 340.1374 . 

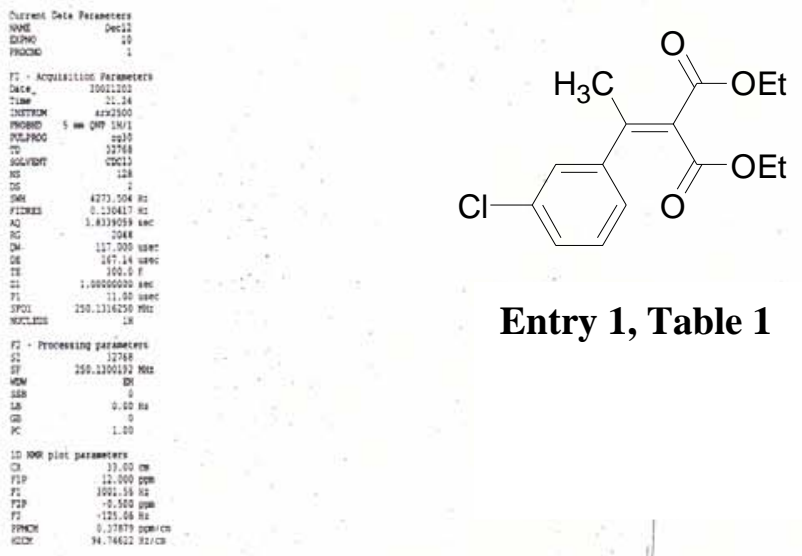

\section{Entry 1, Table 1}

ma

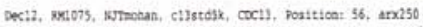

s

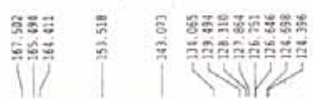

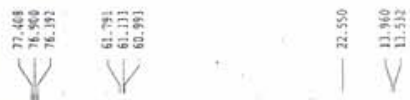

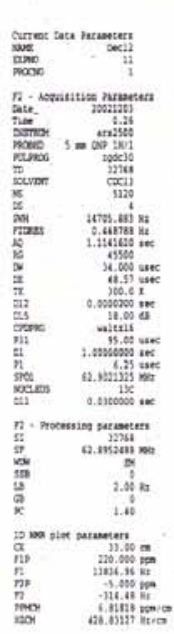

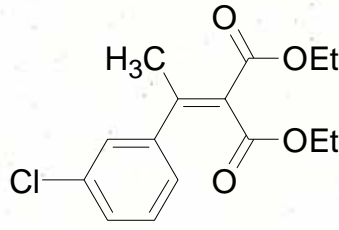

Entry 1, Table 1 


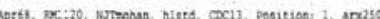

5
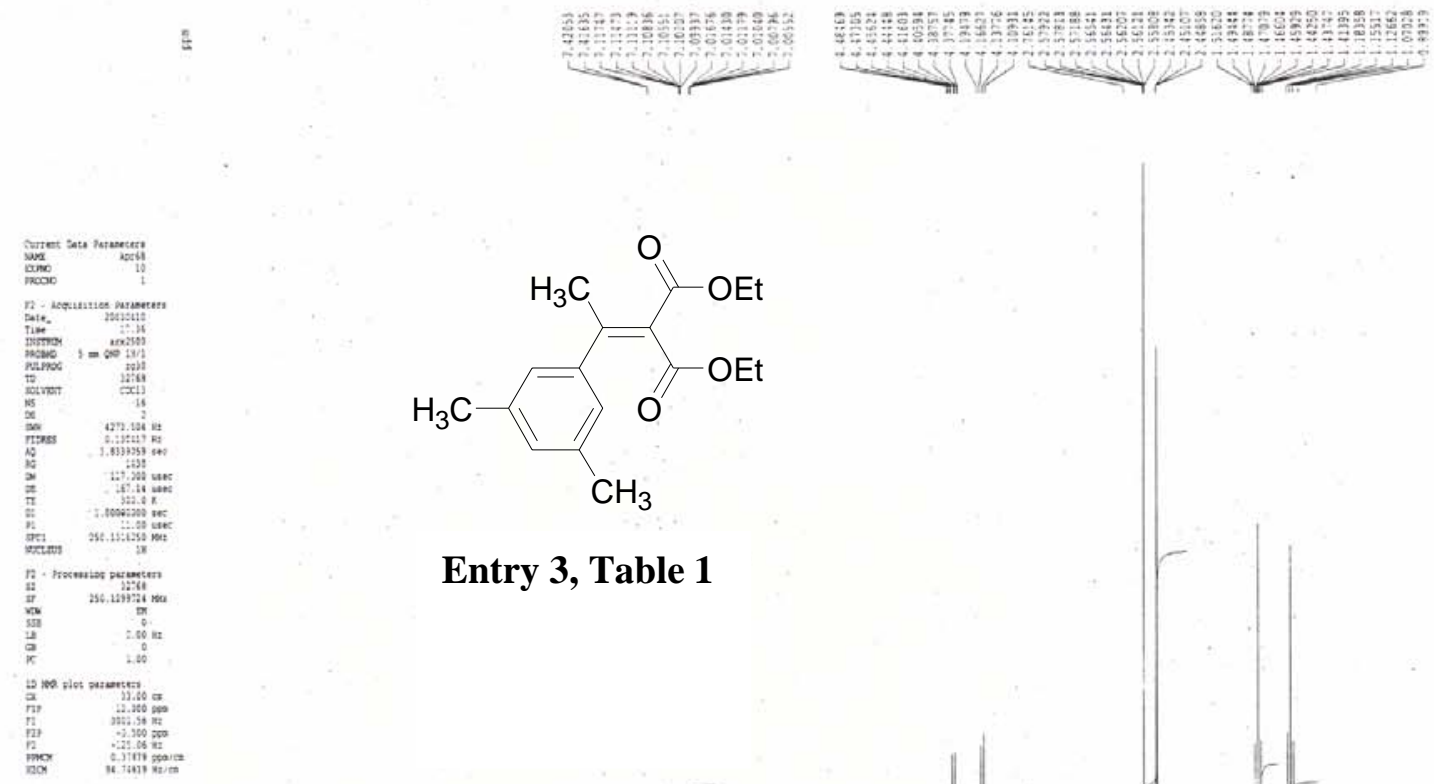

Entry 3, Table 1
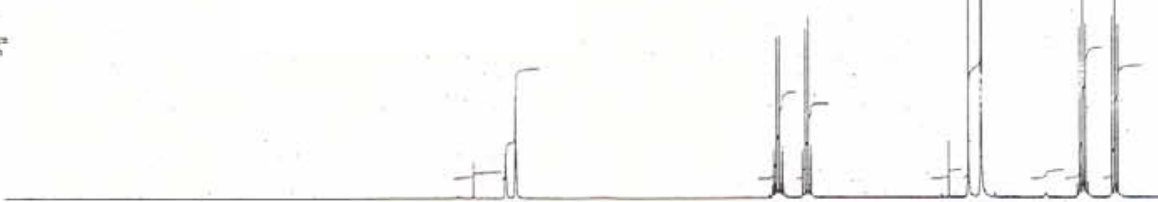

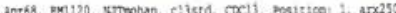

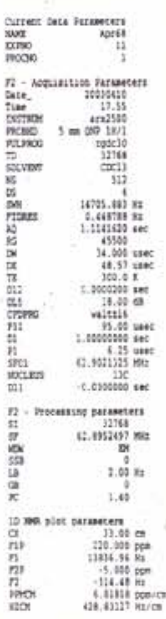

$\mathrm{H}_{3} \mathrm{C}$

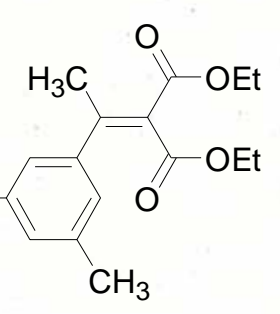

Entry 3, Table 1 


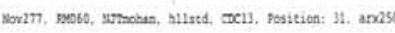
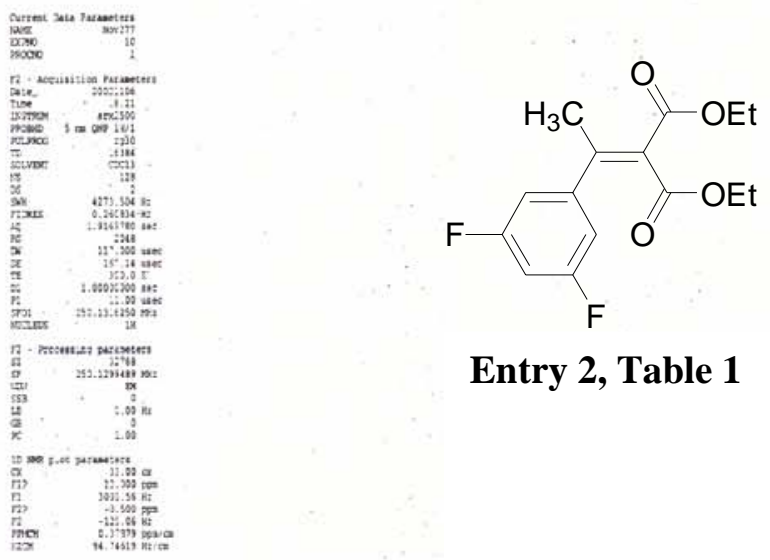

Entry 2, Table 1
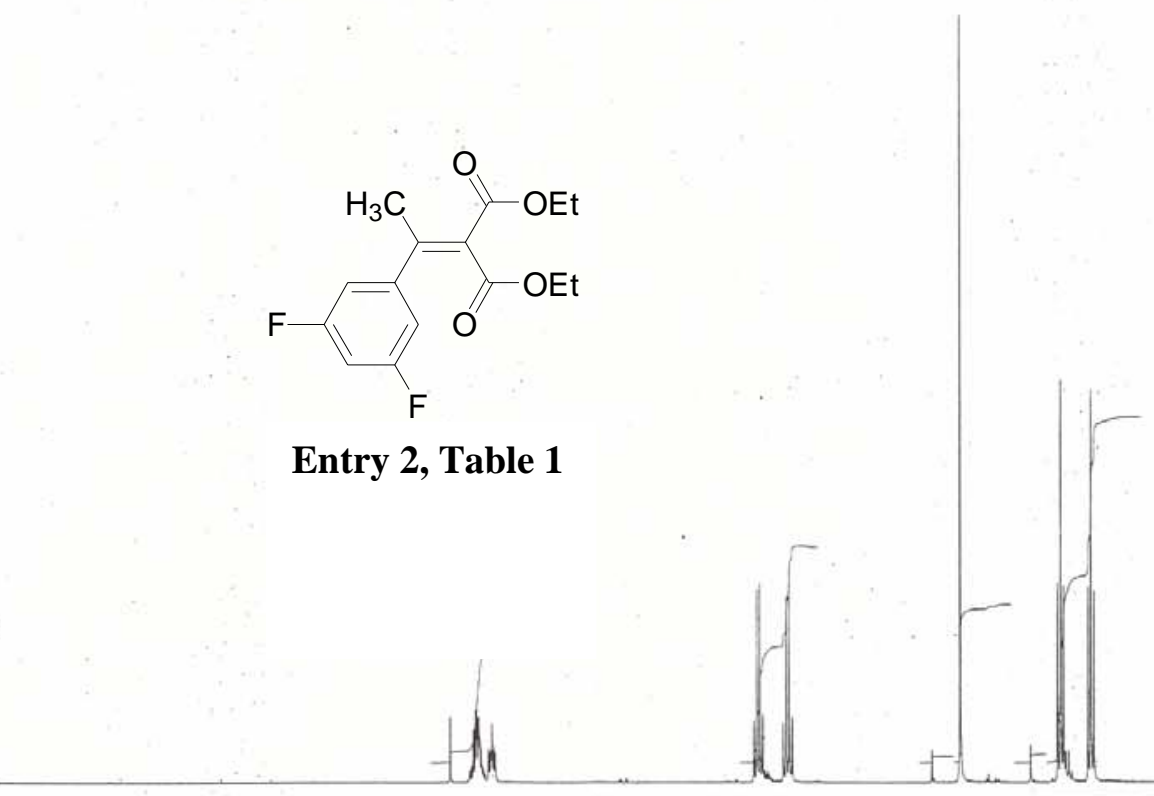

sen 


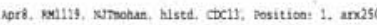
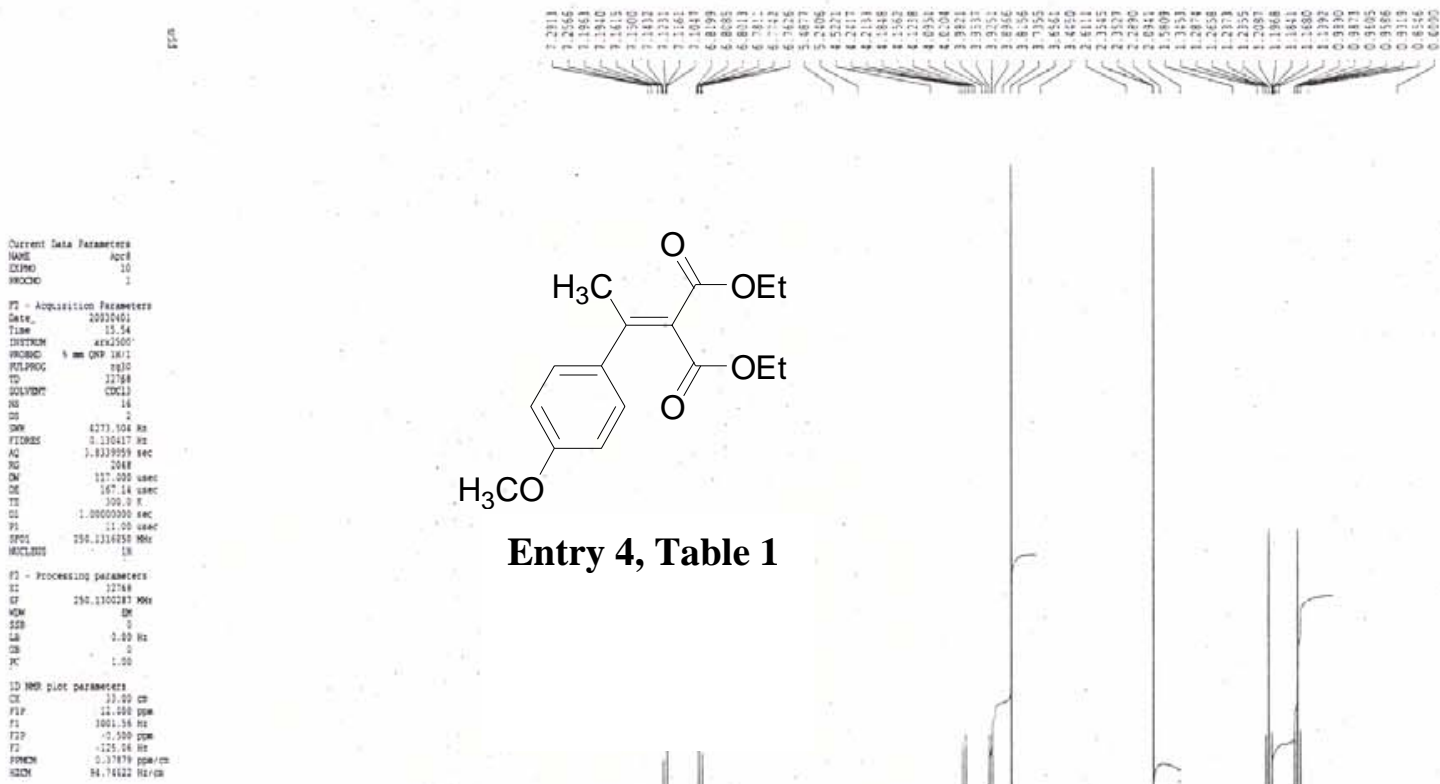

Entry 4, Table 1
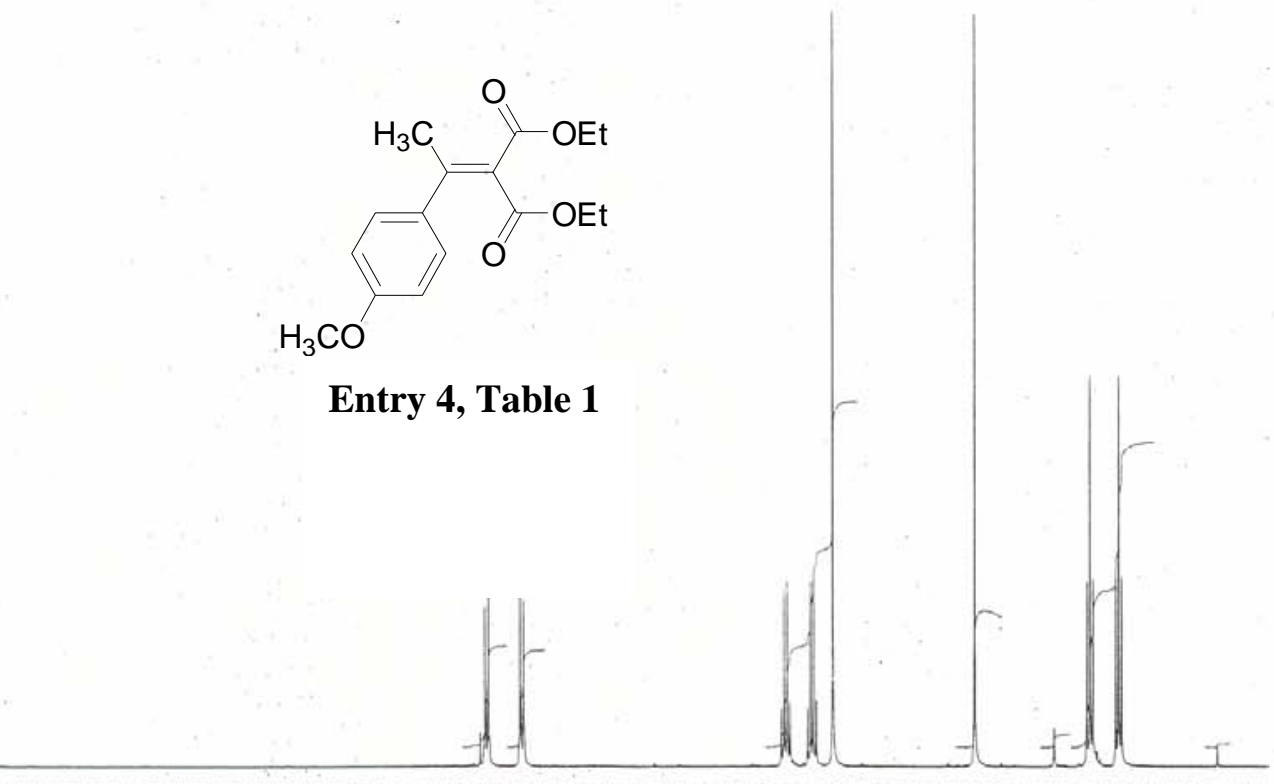

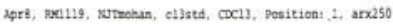

是

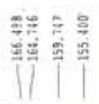

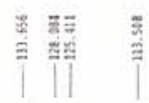

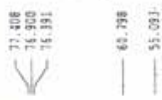

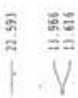
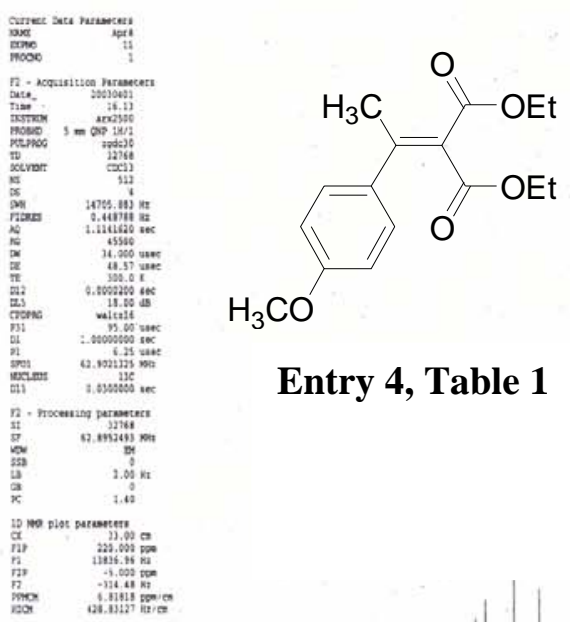

Entry 4, Table 1 


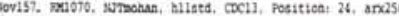
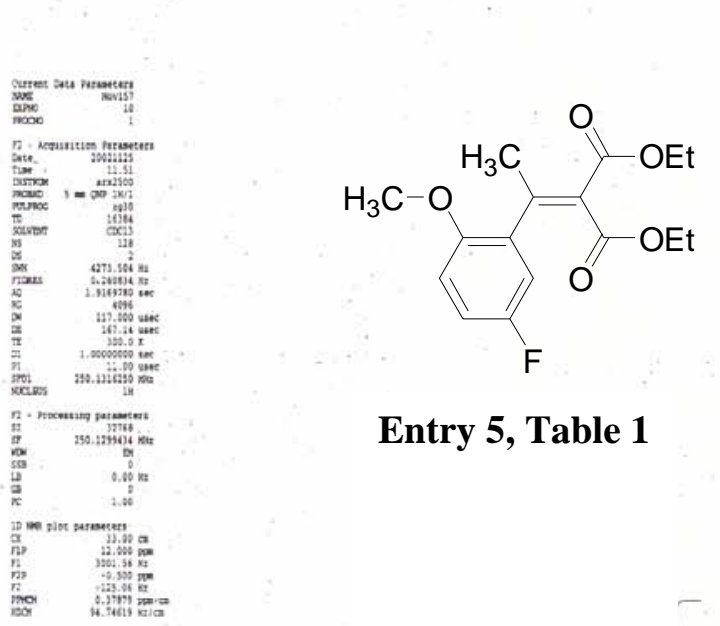

Entry 5, Table 1

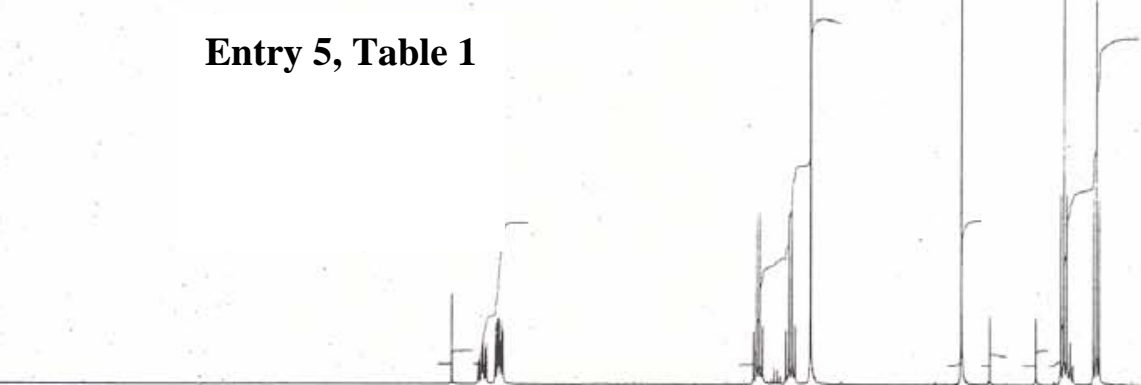

not

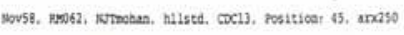

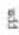
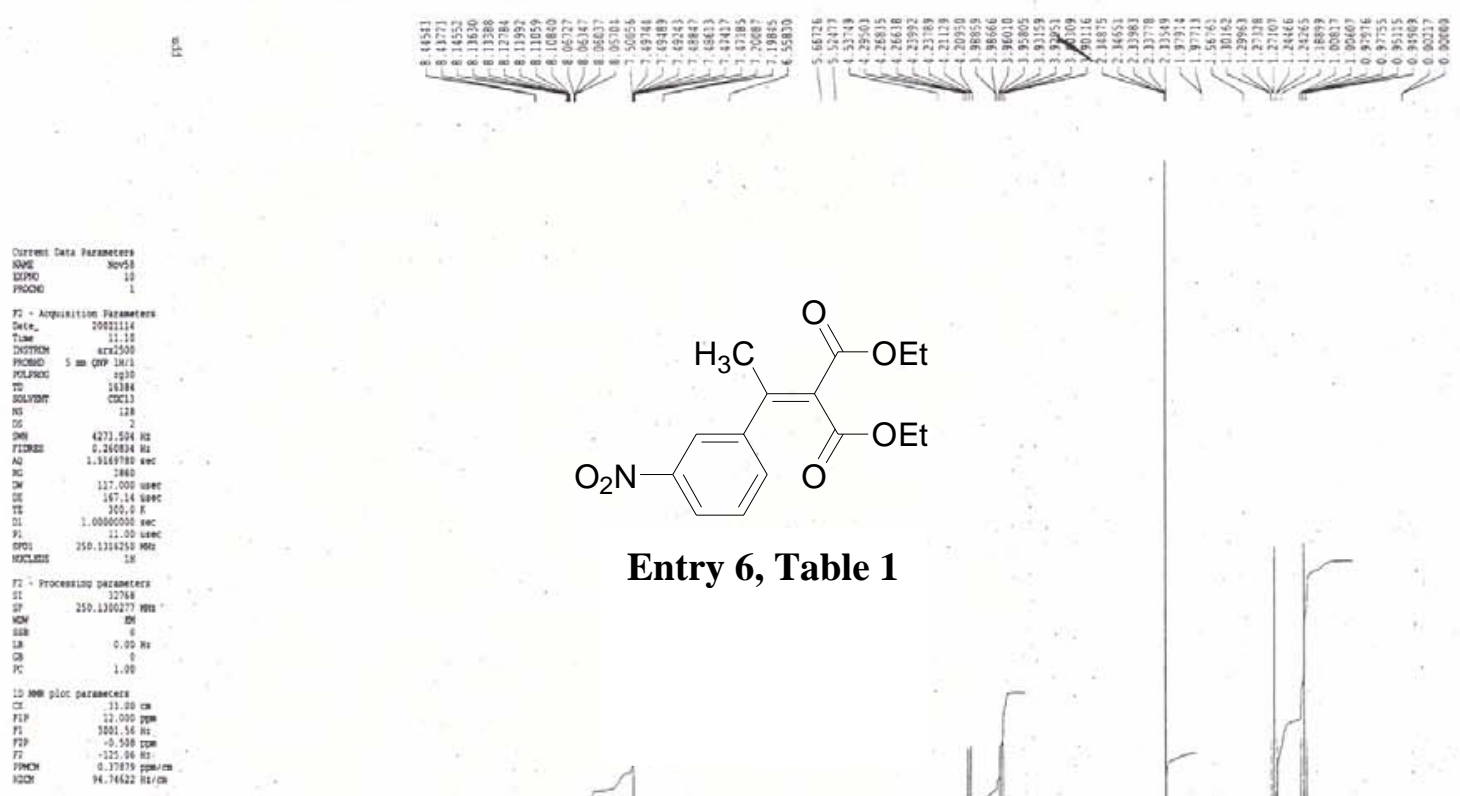

Entry 6, Table 1

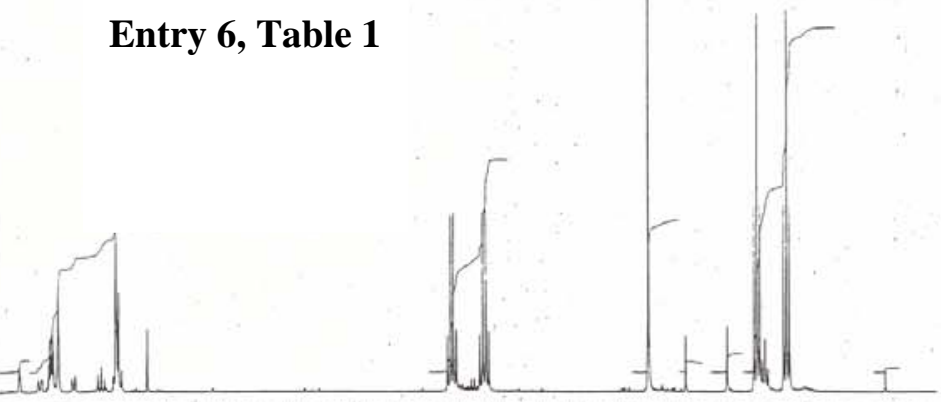




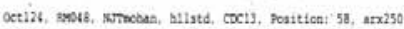
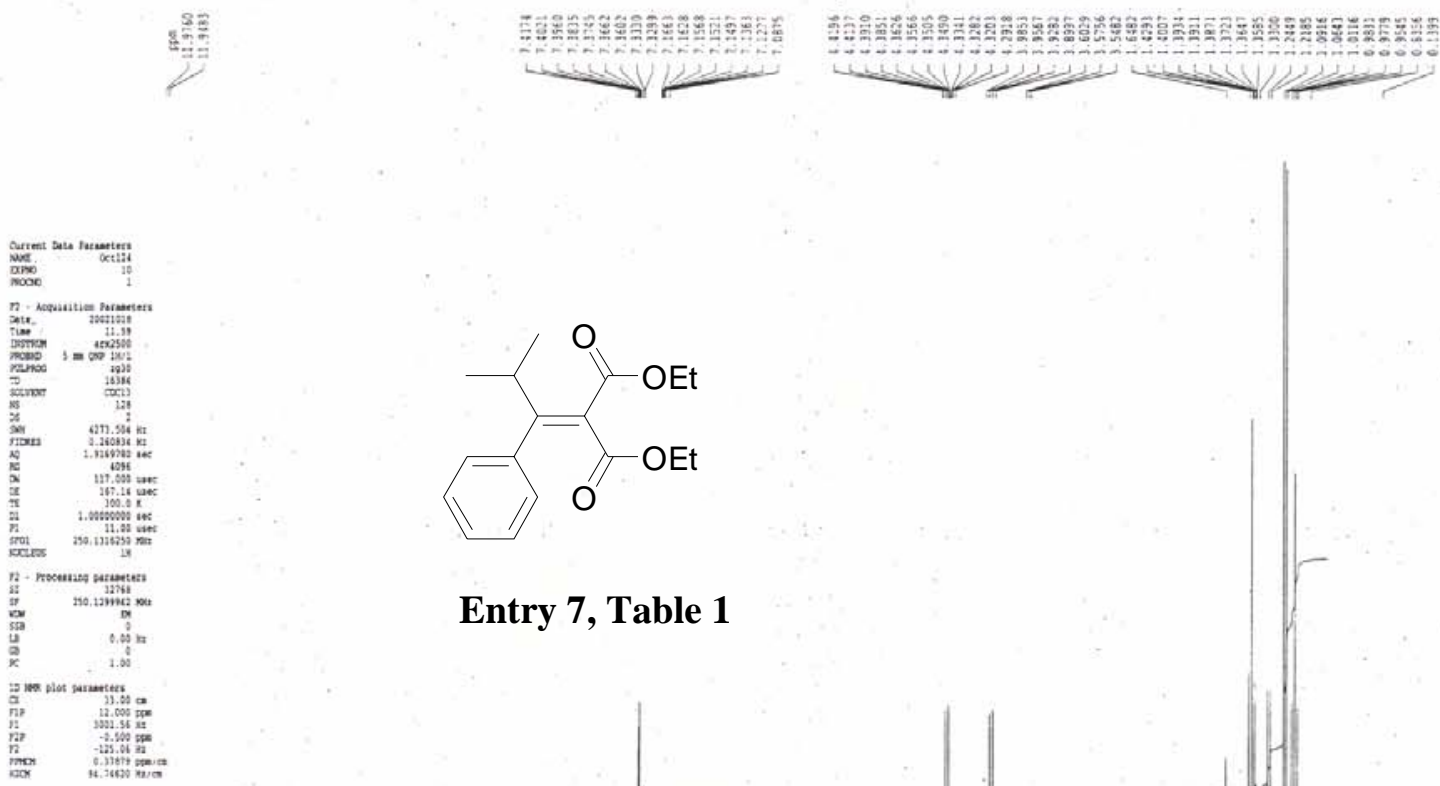

Entry 7, Table 1

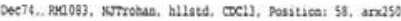
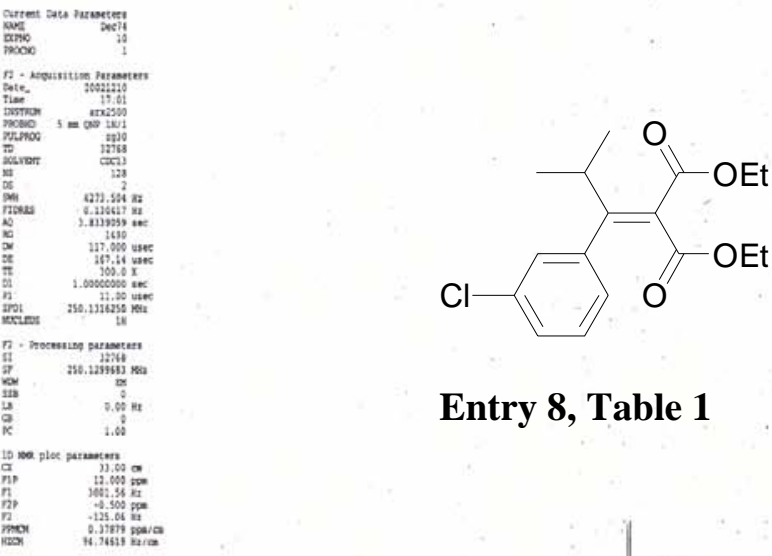

Entry 8, Table 1

.
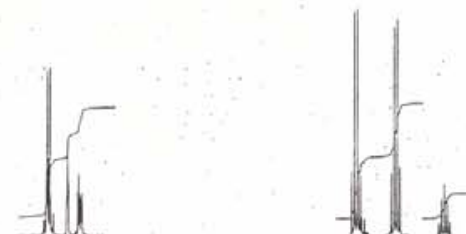


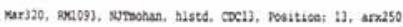

送

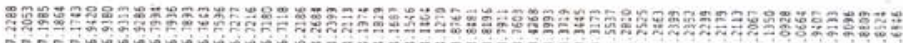

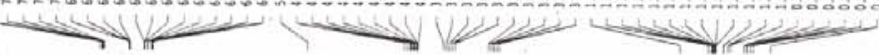
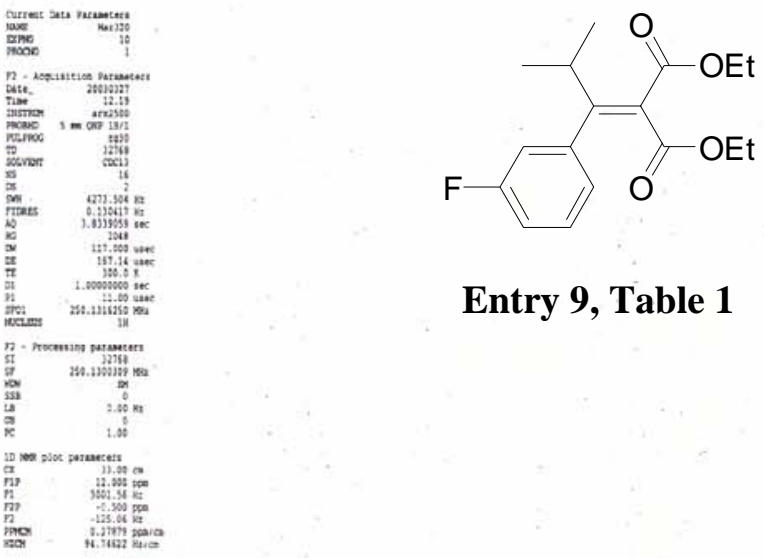

Entry 9, Table 1

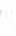
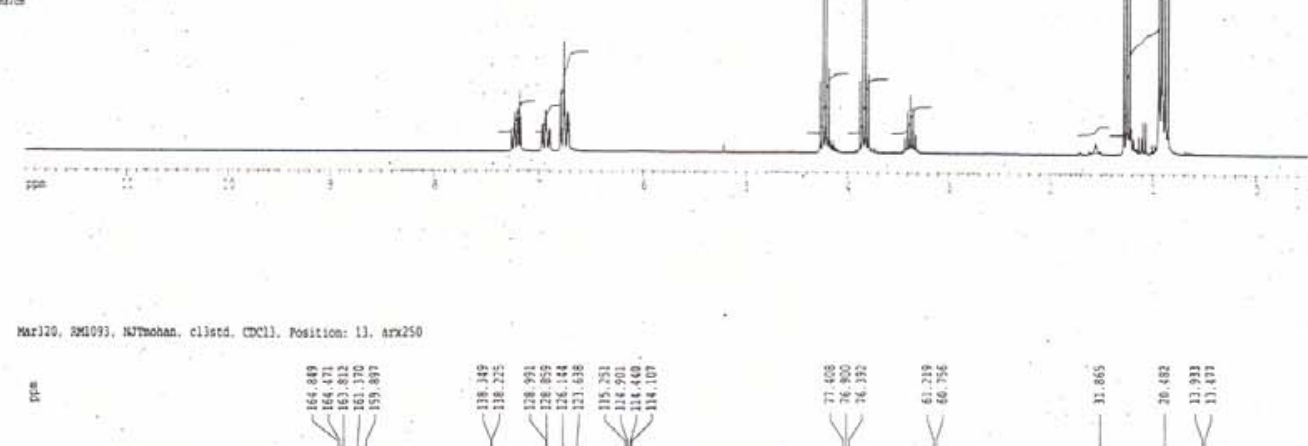

ำ
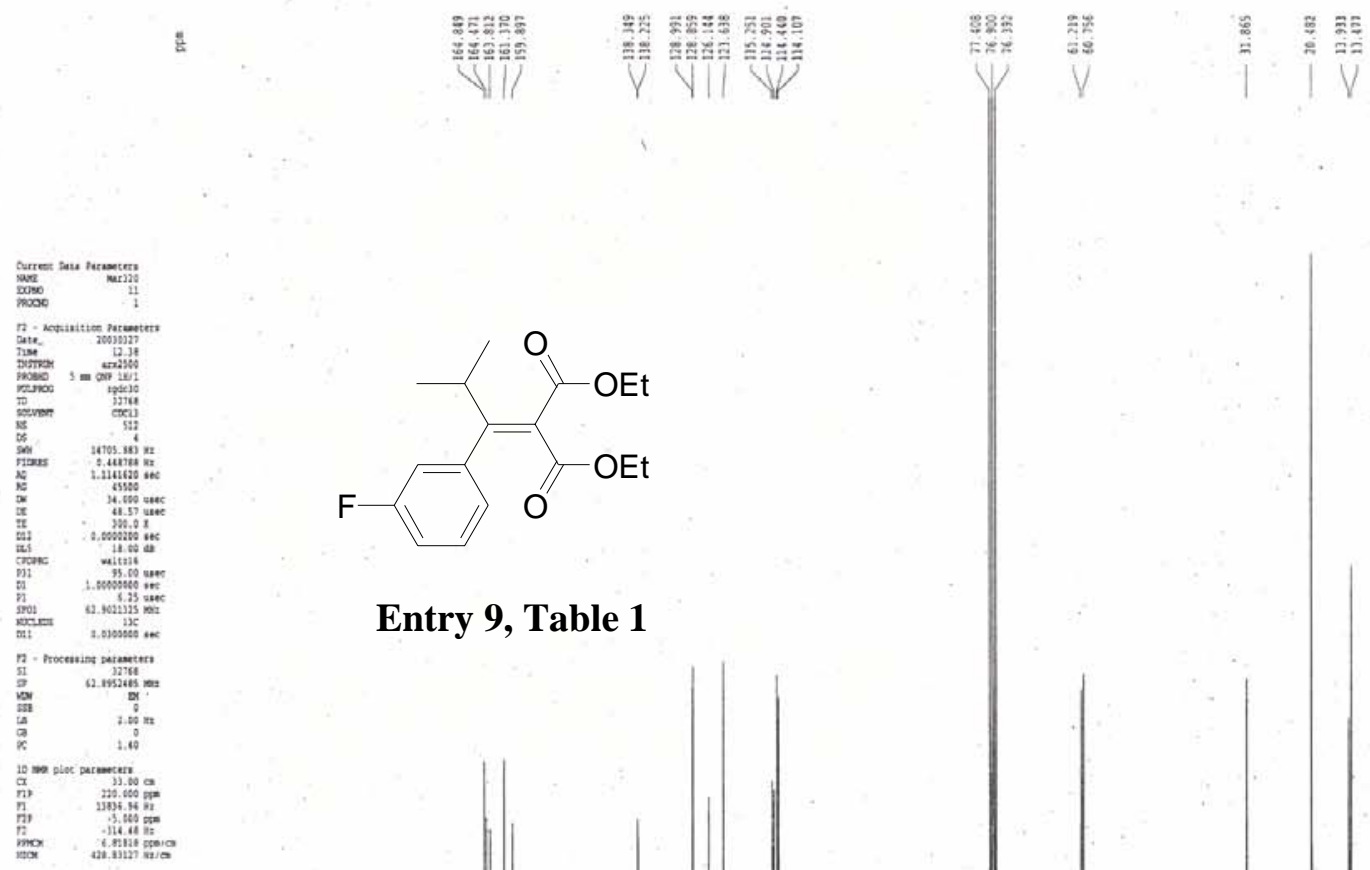

Entry 9, Table 1

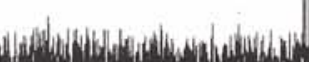




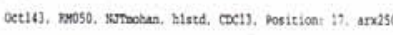

:
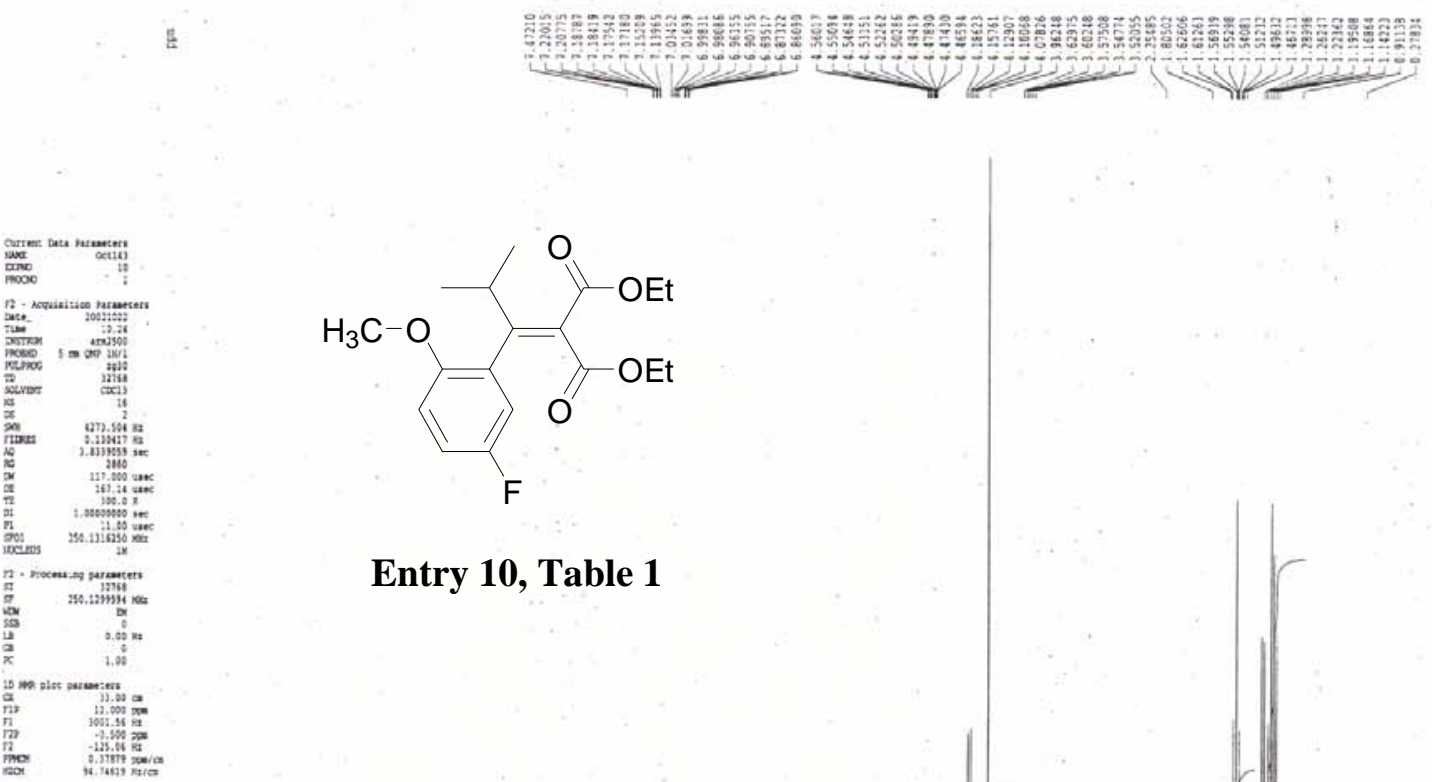

Entry 10, Table 1
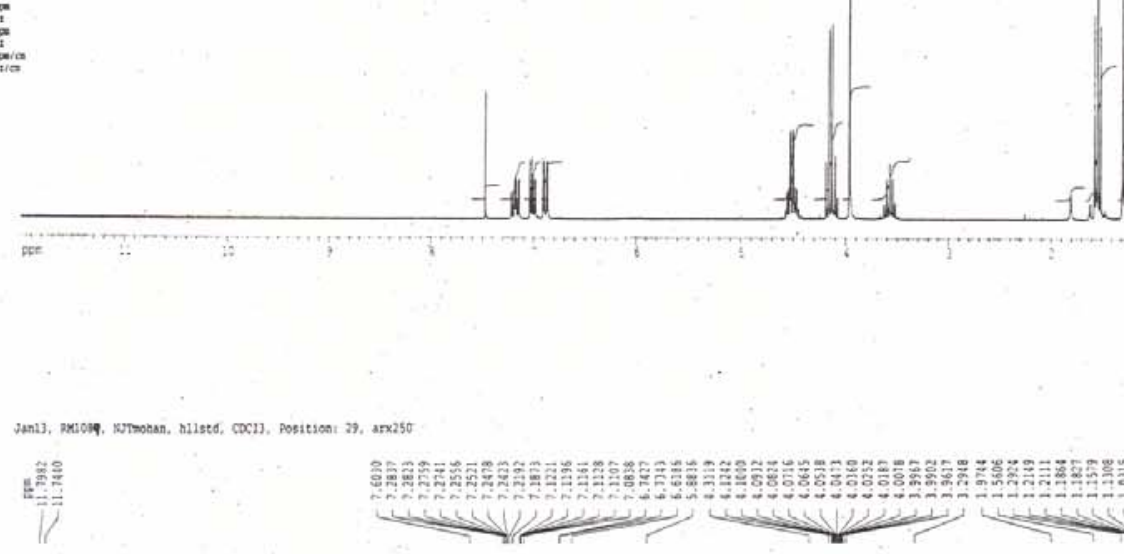

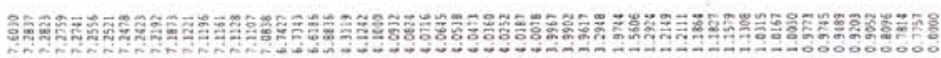
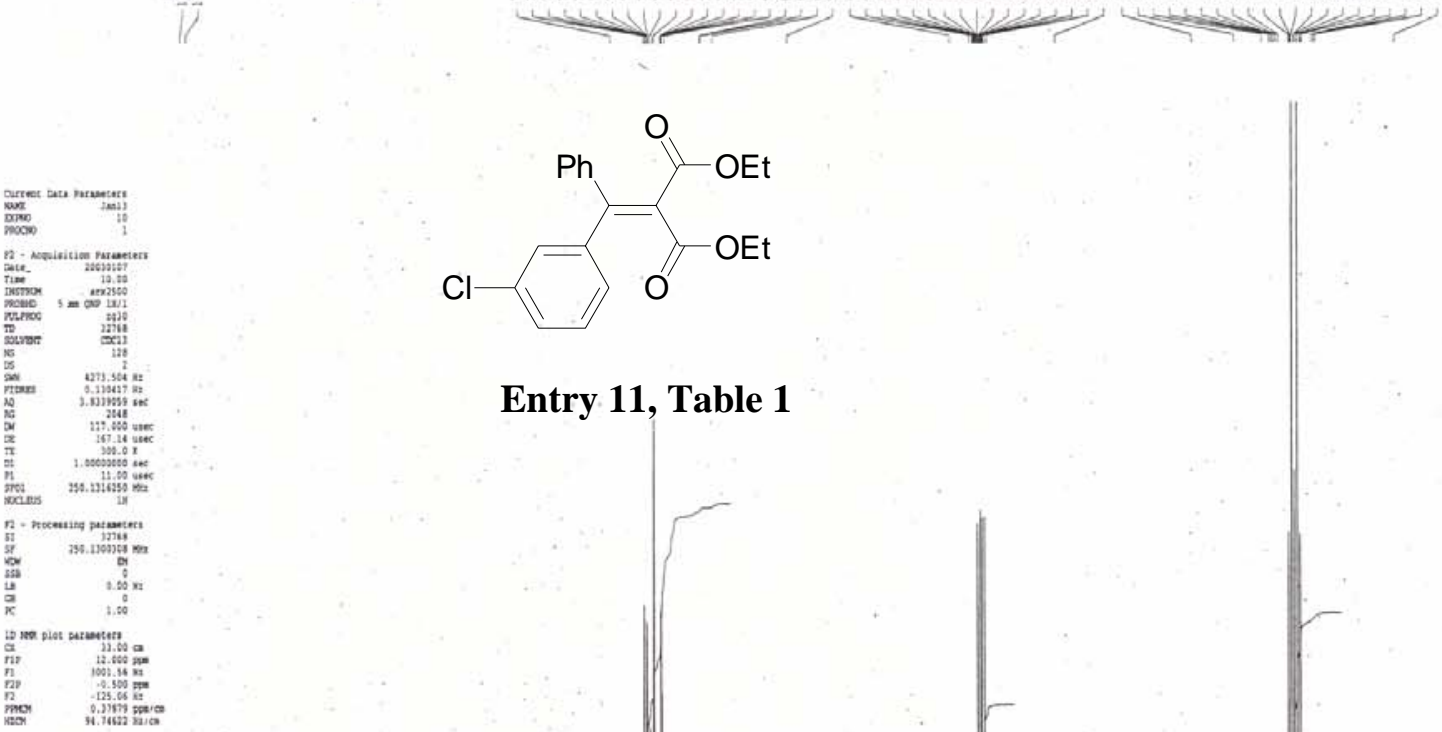

Entry 11, Table 1

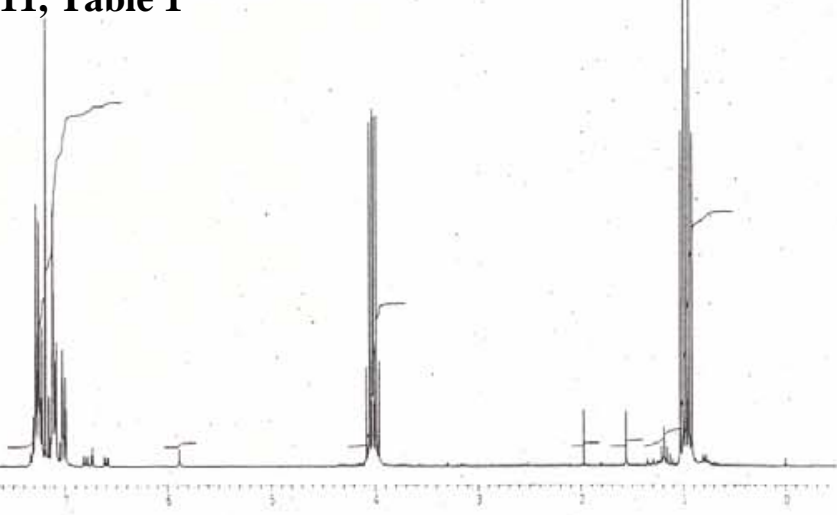



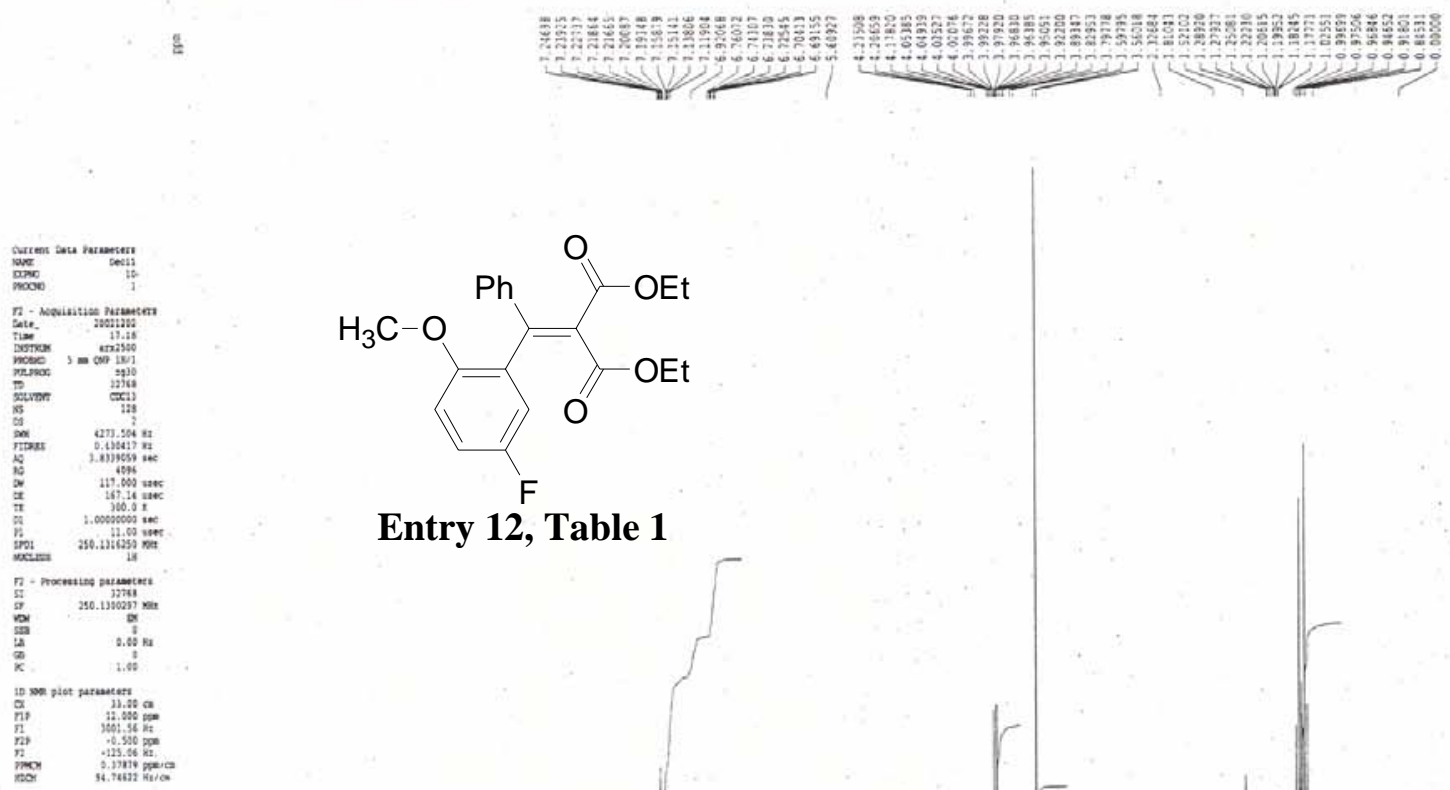

$\mathrm{F}$

Entry 12, Table 1
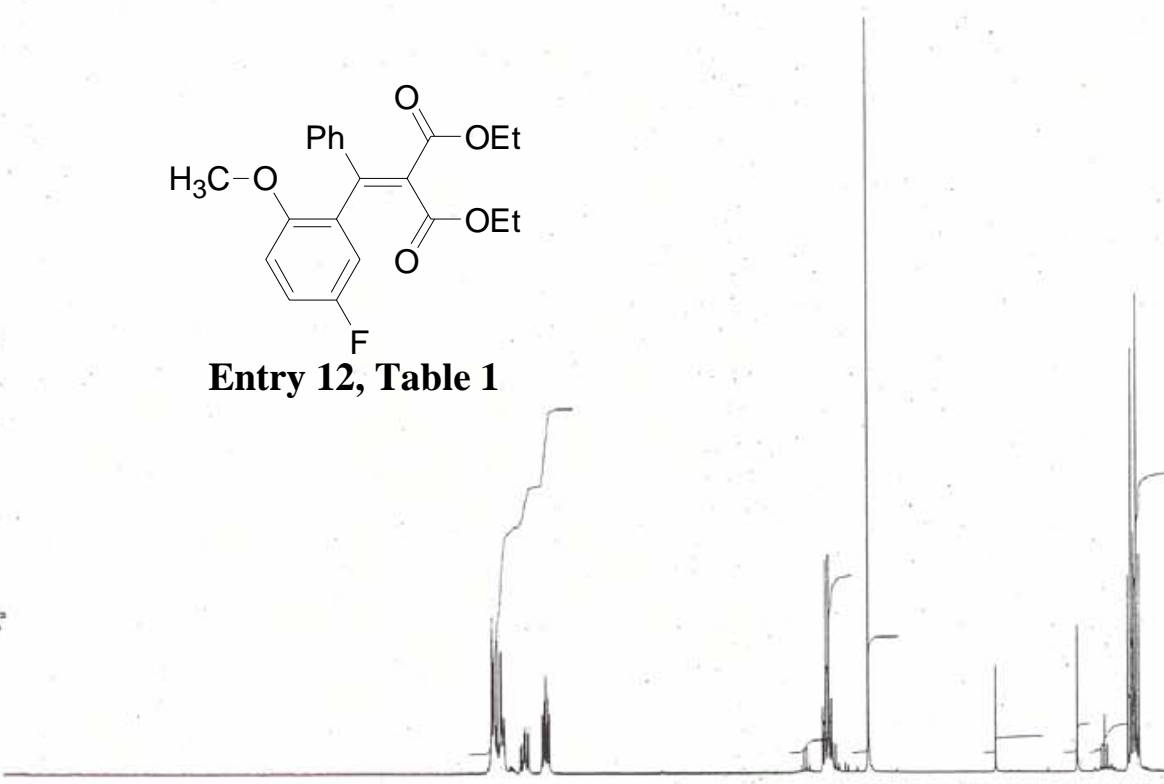

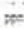

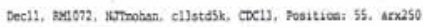

$\$$
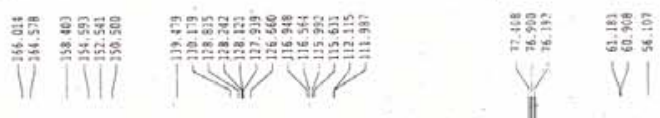

5:
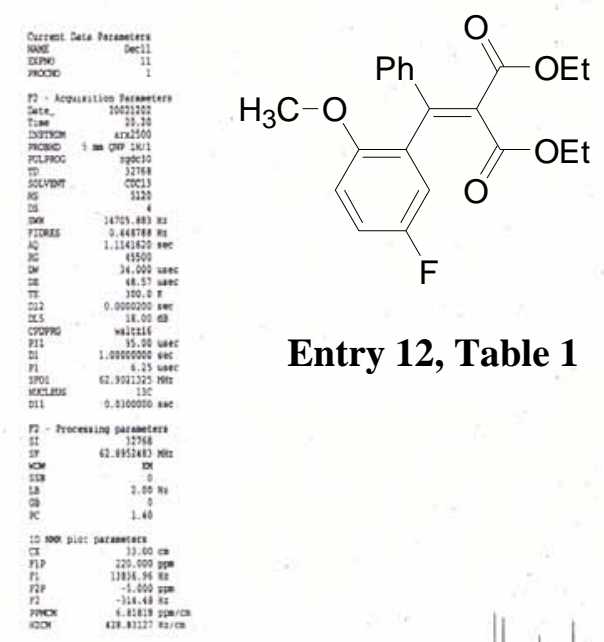

Entry 12, Table 1 


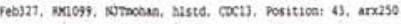
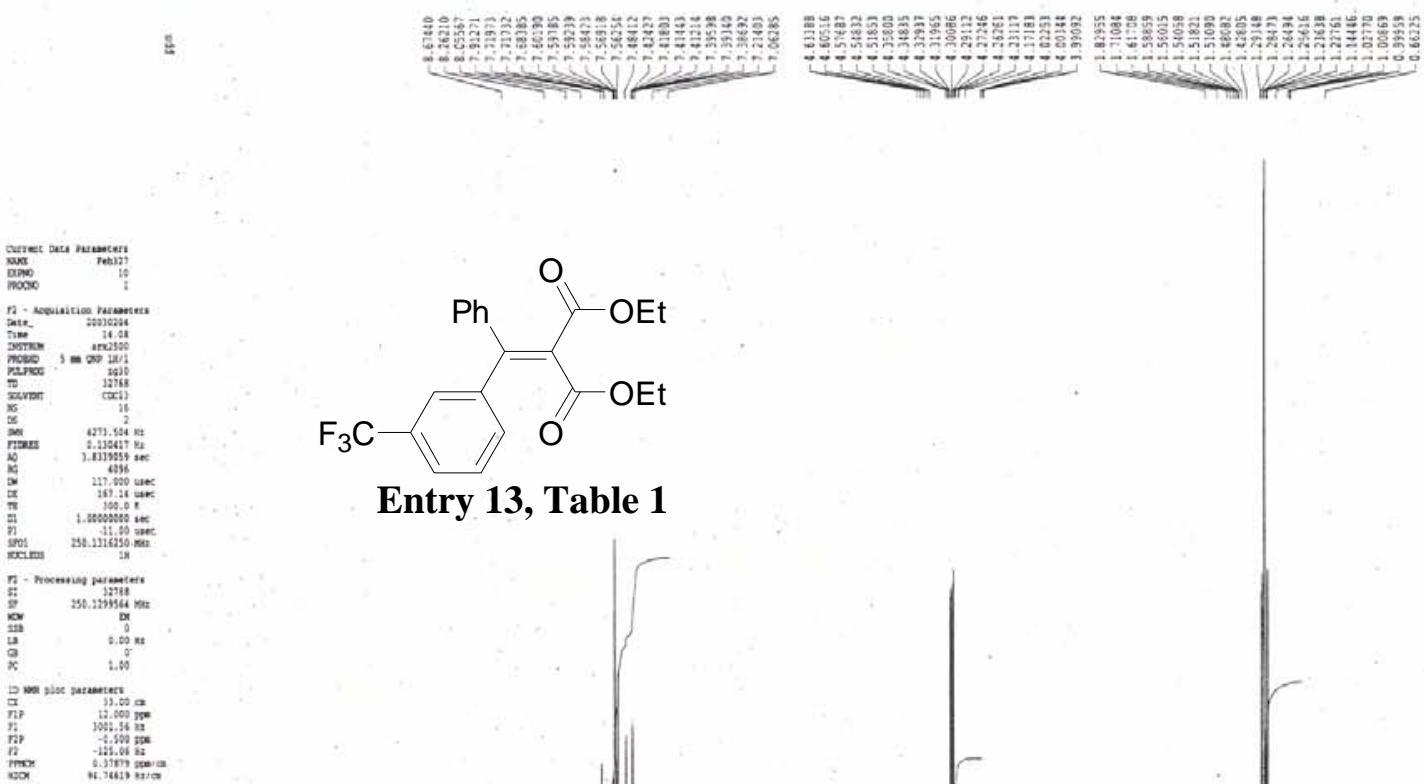

Entry 13, Table 1

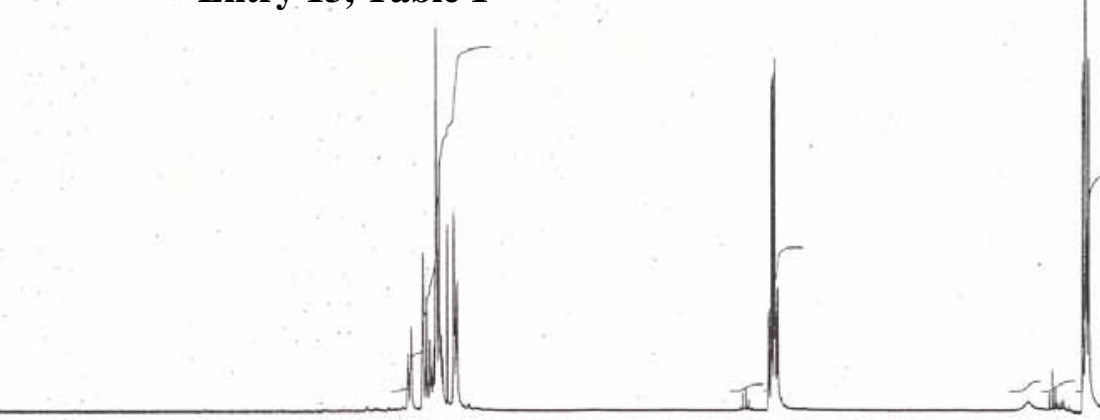

EFe

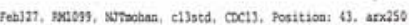

E
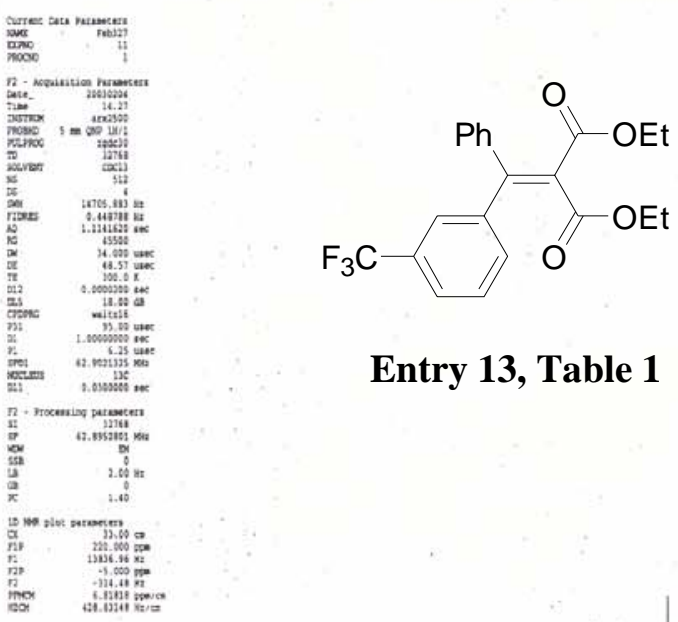

Entry 13, Table 1

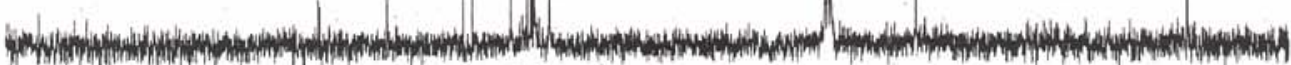



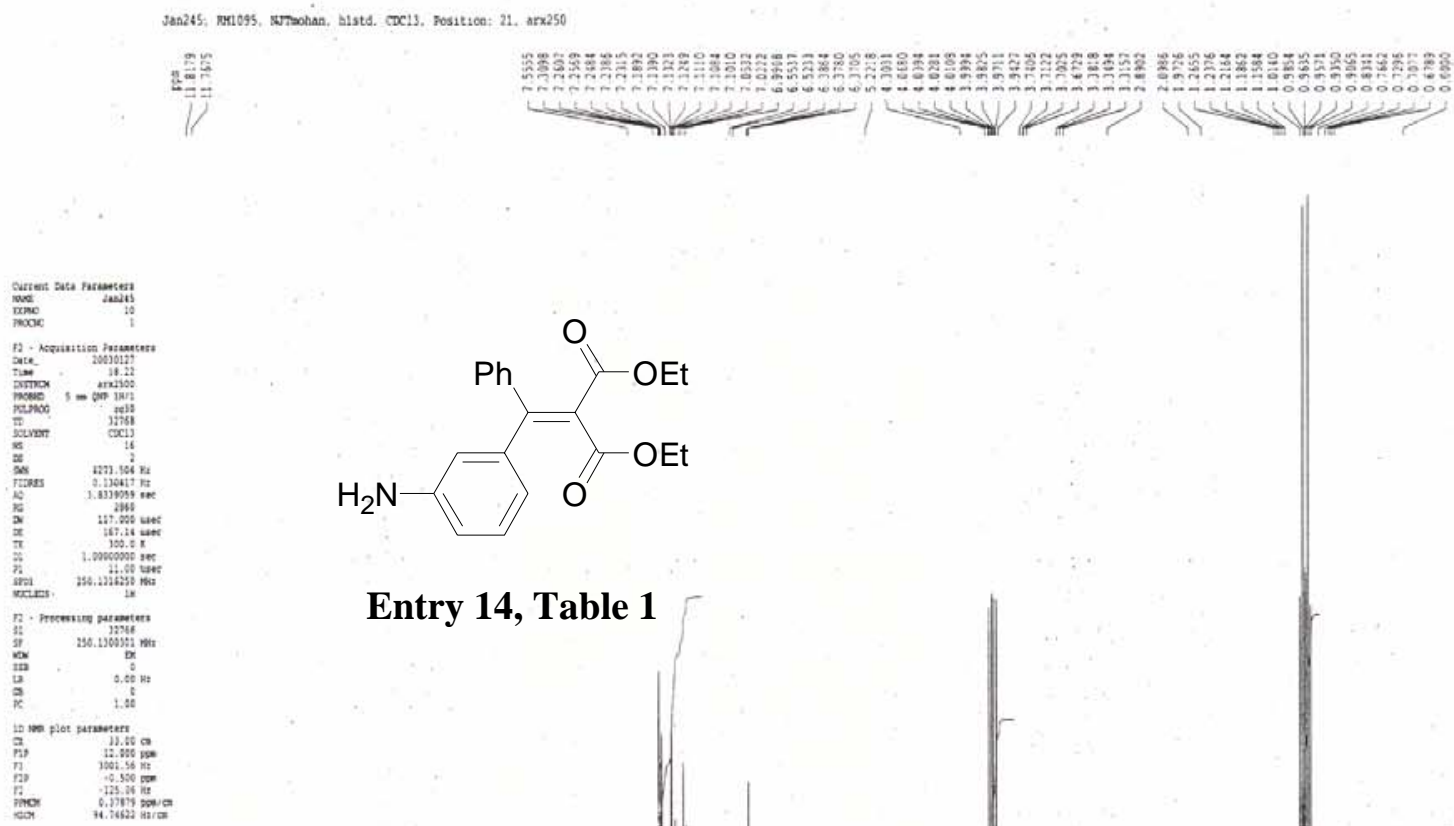

Entry 14, Table 1
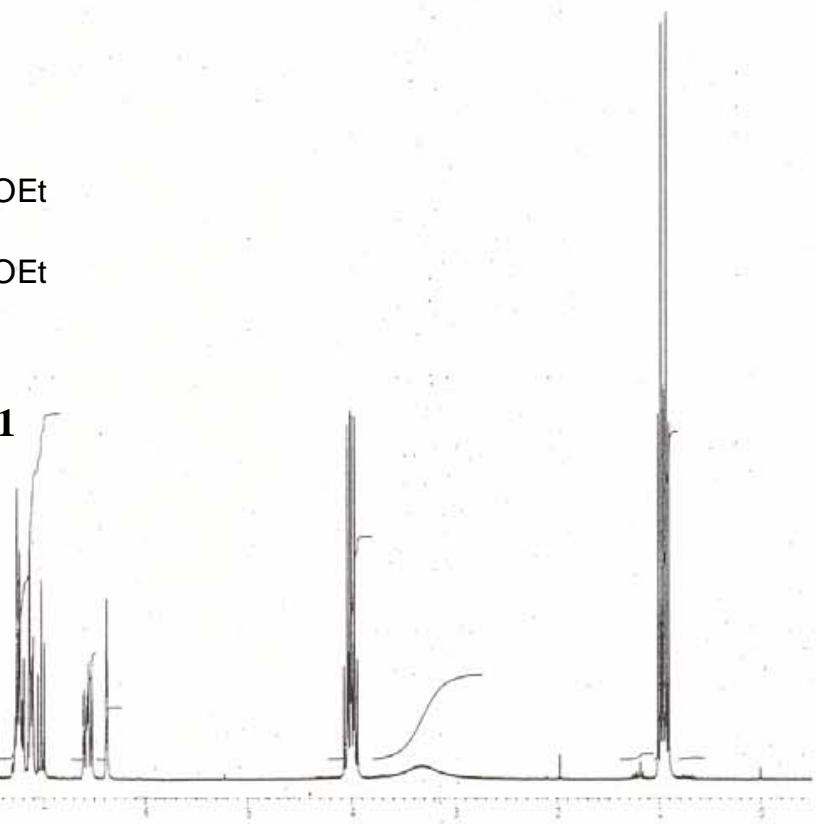

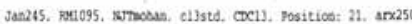

5
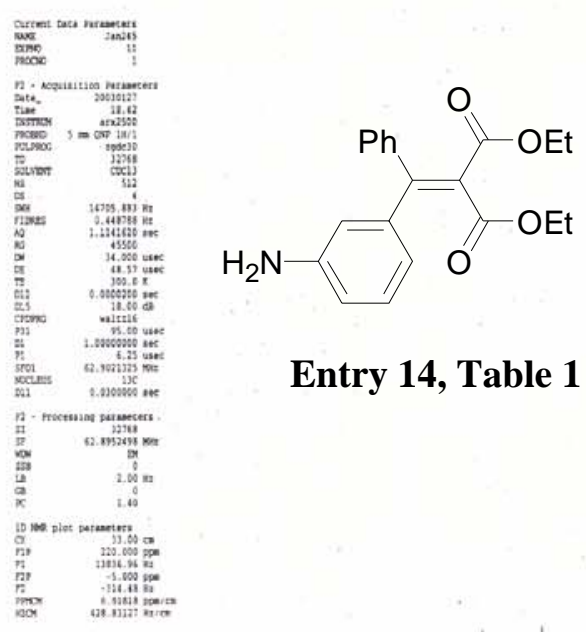

Entry 14, Table 1 


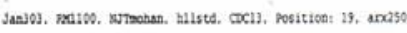

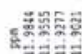

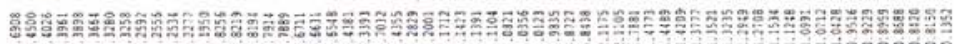

$+$

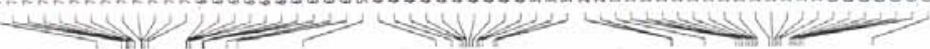

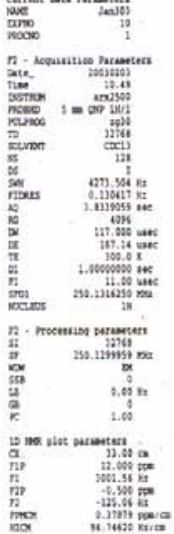

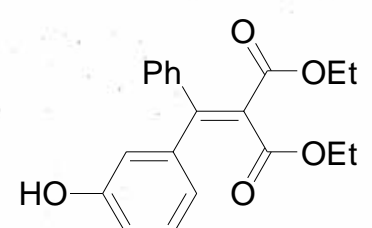

Entry 15, Table 1

告 总

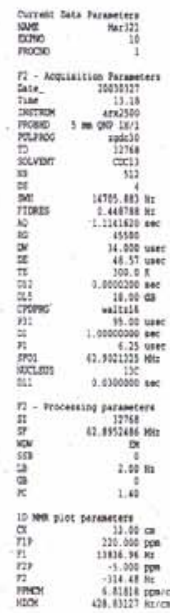

$\mathrm{HO}=\mathrm{O}$

Entry 15, Table 1 